\title{
Central African Republic: Statistical Appendix
}

This Statistical Appendix for the Central African Republic was prepared by a staff team of the International Monetary Fund as background documentation for the periodic consultation with the member country. It is based on the information available at the time it was completed on September 13, 2007. The views expressed in this document are those of the staff team and do not necessarily reflect the views of the government of the Central African Republic or the Executive Board of the IMF.

The policy of publication of staff reports and other documents by the IMF allows for the deletion of market-sensitive information.

To assist the IMF in evaluating the publication policy, reader comments are invited and may be sent by e-mail to publicationpolicy@imf.org.

Copies of this report are available to the public from

International Monetary Fund $\bullet$ Publication Services

700 19th Street, N.W. • Washington, D.C. 20431

Telephone: (202) $6237430 \bullet$ Telefax: (202) 6237201

E-mail: publications@imf.org • Internet: http://www.imf.org

Price: $\$ 18.00$ a copy

\section{International Monetary Fund Washington, D.C.}





\section{INTERNATIONAL MONETARY FUND}

\section{CENTRAL AFRICAN REPUBLIC}

\section{Statistical Appendix}

Prepared by Robert York, Liliana Schumacher, Noriaki Kinoshita, Jean-Claude Nachega Approved by African Department

September 13, 2007

Contents

Page

Tables

1. Gross Domestic Product at Current Prices, 2002-06................................................

2. Gross Domestic Product at Previous Year's Prices, 2002-06 …………………......

3. Gross Domestic Product, Real Growth Rates, 2002-06 ............................................5

4. Supply and Use of Resources at Current Prices, 2002-06 ......................................6

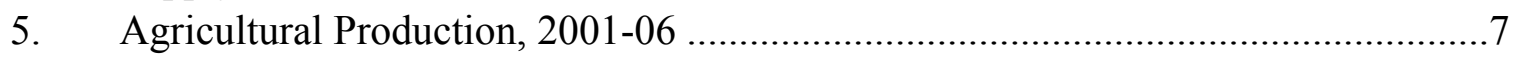

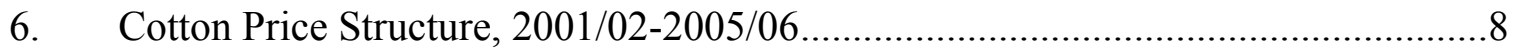

7. Production and Export Volumes of Forestry Products, 2002-06 …………….........9

8. Manufacturing Output, 2002-06 ....................................................................... 10

9. Production and Export of Diamonds and Gold, 2002-06 ......................................11

10. Consumption of Oil Products, 2002-06 ..........................................................12

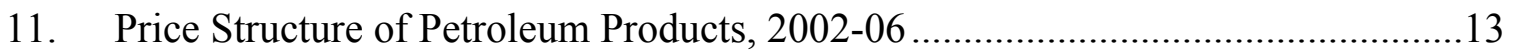

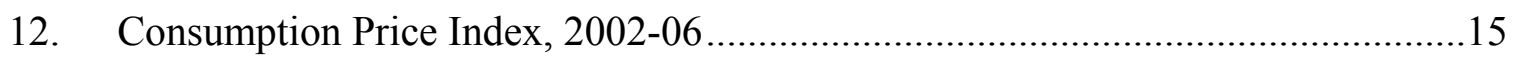

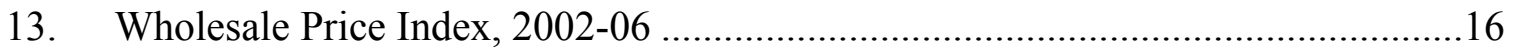

14. Sectoral Composition of the Public Investment Program, 2002-06 …....................17

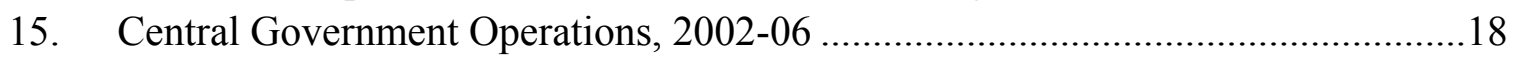

16. Central Government Revenue, 2002-06 ………...............................................19

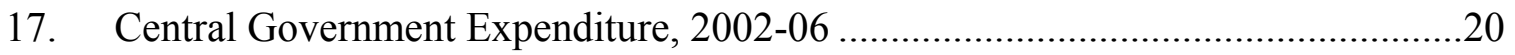

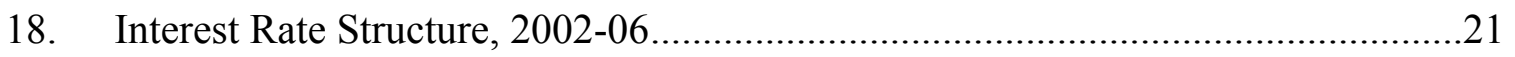

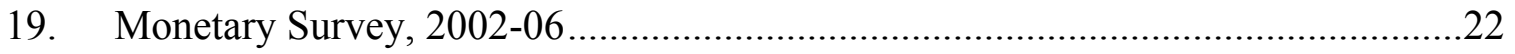

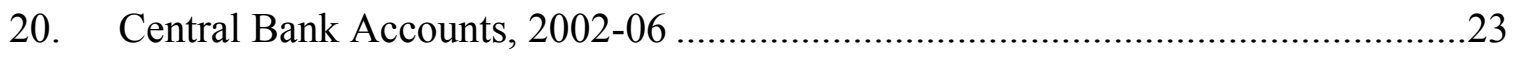

21. Balance Sheet of Commercial Banks, 2002-06 ....................................................24

22. Balance of Payments, 2002-06 (Millions of SDRs) ……………........................25

23. Balance of Payments, 2002-06 (Billions of CFA francs, unless otherwise

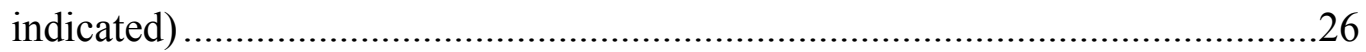

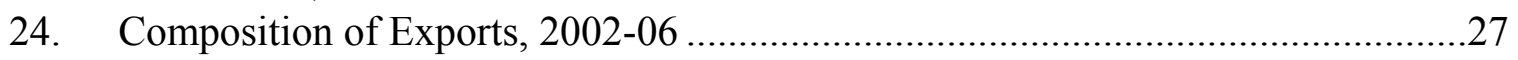


25. Export, Import Indices and Terms of Trade, 2002-06 ........................................28

26. Balance of Payment: Selected Components of Services, Income, and Transfers, 2002-06.

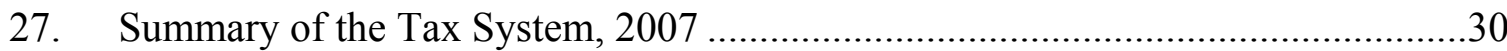


Table 1. Central African Republic: Gross Domestic Product at Current Prices, 2002-06

\begin{tabular}{|c|c|c|c|c|c|}
\hline & 2002 & 2003 & 2004 & 2005 & 2006 \\
\hline & \multicolumn{5}{|c|}{ (In billions of CFA francs) } \\
\hline Primary sector & 370.4 & 376.8 & 372.3 & 384.5 & 421.8 \\
\hline Agriculture & 197.4 & 208.8 & 203.0 & 210.1 & 222.7 \\
\hline Subsistence & 193.2 & 206.3 & 200.5 & 207.8 & 219.8 \\
\hline Cash crops & 4.2 & 2.6 & 2.6 & 2.4 & 2.9 \\
\hline Livestock & 80.7 & 82.9 & 80.4 & 83.3 & 86.3 \\
\hline Hunting and fishing & 38.6 & 40.3 & 41.3 & 43.2 & 44.8 \\
\hline Hunting & 24.9 & 26.0 & 26.7 & 27.8 & 28.9 \\
\hline Fishing & 13.7 & 14.3 & 14.7 & 15.4 & 15.9 \\
\hline Forestry & 53.7 & 44.7 & 47.6 & 47.9 & 68.1 \\
\hline Logs & 32.4 & 29.0 & 30.4 & 28.1 & 43.1 \\
\hline Other & 21.3 & 15.7 & 17.1 & 19.8 & 25.0 \\
\hline Secondary sector & 104.1 & 98.8 & 98.9 & 106.1 & 115.3 \\
\hline Manufacturing industry & 21.5 & 16.4 & 16.3 & 17.7 & 18.0 \\
\hline Mining & 47.7 & 46.1 & 46.5 & 50.2 & 55.8 \\
\hline Public utilities & 5.2 & 5.4 & 5.6 & 5.8 & 6.4 \\
\hline Construction & 29.7 & 30.9 & 30.6 & 32.4 & 35.2 \\
\hline Tertiary sector & 207.5 & 187.9 & 186.7 & 196.5 & 200.9 \\
\hline Merchant services & 142.5 & 136.4 & 138.3 & 133.7 & 142.7 \\
\hline Transport and communications & 27.9 & 26.6 & 27.7 & 29.1 & 35.4 \\
\hline Commerce & 75.7 & 72.3 & 75.3 & 79.9 & 86.5 \\
\hline Other & 39.0 & 37.6 & 35.3 & 24.6 & 20.8 \\
\hline Nonmerchant services & 65.0 & 51.5 & 48.4 & 62.9 & 58.2 \\
\hline Central government & 45.1 & 47.0 & 42.5 & 42.8 & 40.6 \\
\hline Technical assistance & 19.9 & 4.5 & 5.9 & 20.0 & 17.7 \\
\hline GDP at factor costs & 682.1 & 663.5 & 658.0 & 687.1 & 738.1 \\
\hline Indirect taxes & 36.5 & 23.6 & 25.5 & 28.9 & 31.1 \\
\hline Customs duties & 7.6 & 7.7 & 7.1 & 7.0 & 11.7 \\
\hline GDP at market prices & 726.2 & 694.7 & 690.6 & 723.0 & 781.0 \\
\hline
\end{tabular}

Sources: C.A.R. authorities; and Fund staff estimates. 
Table 2. Central African Republic: Gross Domestic Product at Previous Year's Prices, 2002-06 ${ }^{1}$

\begin{tabular}{|c|c|c|c|c|c|}
\hline & 2002 & 2003 & 2004 & 2005 & 2006 \\
\hline & \multicolumn{5}{|c|}{ (In billions of CFA francs) } \\
\hline Primary sector & 357.9 & 344.0 & 351.1 & 352.2 & 368.6 \\
\hline Agriculture & 186.0 & 186.4 & 190.7 & 192.6 & 198.2 \\
\hline Subsistence & 180.9 & 183.6 & 187.6 & 189.9 & 195.4 \\
\hline Cash crops & 5.1 & 2.8 & 3.1 & 2.8 & 2.8 \\
\hline Livestock & 73.8 & 74.3 & 75.8 & 76.6 & 77.2 \\
\hline Hunting and fishing & 35.3 & 36.1 & 37.0 & 37.6 & 38.0 \\
\hline Hunting & 22.7 & 23.3 & 23.9 & 24.2 & 24.5 \\
\hline Fishing & 12.5 & 12.8 & 13.1 & 13.4 & 13.5 \\
\hline Forestry & 62.9 & 47.2 & 47.6 & 45.4 & 55.2 \\
\hline Logs & 37.7 & 29.3 & 29.2 & 26.3 & 34.2 \\
\hline Others & 25.2 & 17.9 & 18.4 & 19.1 & 21.0 \\
\hline Secondary sector & 100.5 & 92.4 & 94.8 & 99.3 & 105.3 \\
\hline Manufacturing industry & 23.2 & 18.2 & 19.9 & 21.4 & 23.1 \\
\hline Mining & 44.4 & 41.3 & 41.7 & 43.8 & 46.6 \\
\hline Public utilities & 5.3 & 5.3 & 5.3 & 5.4 & 5.7 \\
\hline Construction & 27.6 & 27.6 & 27.9 & 28.7 & 29.9 \\
\hline Tertiary sector & 185.1 & 166.9 & 164.0 & 170.4 & 170.2 \\
\hline Merchant services & 130.2 & 124.8 & 123.5 & 129.5 & 132.7 \\
\hline Transport and communications & 24.9 & 23.5 & 24.0 & 25.0 & 29.4 \\
\hline Commerce & 67.6 & 63.9 & 65.2 & 68.5 & 71.9 \\
\hline Others & 37.7 & 37.4 & 34.4 & 36.0 & 31.3 \\
\hline Nonmerchant services & 55.0 & 42.1 & 40.5 & 40.9 & 37.6 \\
\hline Central government & 38.1 & 38.5 & 35.5 & 34.8 & 32.3 \\
\hline Technical assistance & 16.8 & 3.7 & 5.0 & 6.1 & 5.3 \\
\hline GDP at factor costs & 643.5 & 603.4 & 610.0 & 621.9 & 644.2 \\
\hline Indirect taxes & 30.9 & 19.3 & 21.4 & 23.6 & 24.5 \\
\hline Customs duties & 6.5 & 6.3 & 6.0 & 5.7 & 9.2 \\
\hline GDP at market prices & 680.9 & 628.9 & 637.3 & 651.3 & 677.9 \\
\hline
\end{tabular}

Sources: C.A.R. authorities; and Fund staff estimates.

${ }^{1}$ Since the base year (1985) is outdated and being updated, the C.A.R. authorities compute real GDP growth by comparing output at previous year prices (Table 1) with previous year's output at current prices. 
Table 3. Central African Republic: Gross Domestic Product, Real Growth Rates, 2002-06 ${ }^{1}$

\begin{tabular}{|c|c|c|c|c|c|}
\hline & 2002 & 2003 & 2004 & 2005 & 2006 \\
\hline & \multicolumn{4}{|c|}{ (Annual percentage changes) } & \\
\hline Primary sector & -0.8 & -3.9 & 2.1 & 0.3 & 4.7 \\
\hline Agriculture & -0.5 & 0.2 & 2.3 & 1.0 & 2.9 \\
\hline Subsistence & 2.0 & 1.5 & 2.2 & 1.2 & 2.9 \\
\hline Cash crops & -46.8 & -44.8 & 10.6 & -11.1 & 2.6 \\
\hline Livestock & 0.5 & 0.7 & 2.0 & 1.1 & 0.8 \\
\hline Hunting and fishing & 2.6 & 2.5 & 2.5 & 1.5 & 1.0 \\
\hline Hunting & 2.6 & 2.5 & 2.5 & 1.4 & 1.0 \\
\hline Fishing & 2.6 & 2.5 & 2.5 & 1.7 & 1.0 \\
\hline Forestry & -5.1 & -25.0 & 0.8 & -4.6 & 21.7 \\
\hline Logs & -1.1 & -22.3 & -0.5 & -9.8 & 30.0 \\
\hline Other & -10.6 & -29.0 & 2.9 & 3.7 & 10.3 \\
\hline Secondary sector & -3.4 & -8.0 & 2.6 & 4.7 & 6.0 \\
\hline Manufacturing industry & -9.5 & -21.2 & 9.0 & 7.5 & 8.0 \\
\hline Mining & -2.5 & -7.0 & 1.0 & 5.0 & 6.5 \\
\hline Public utilities & 1.3 & -1.1 & 1.0 & 1.0 & 5.5 \\
\hline Construction & 0.0 & 0.0 & 1.0 & 3.0 & 4.0 \\
\hline Tertiary sector & -0.6 & -9.8 & -1.7 & 3.9 & -0.1 \\
\hline Merchant services & 0.3 & -4.1 & -1.0 & 4.8 & 2.4 \\
\hline Transport and communications & 0.7 & -5.5 & 2.0 & 4.1 & 17.9 \\
\hline Commerce & -2.5 & -5.5 & 2.0 & 5.1 & 5.0 \\
\hline Other & 5.6 & -0.7 & -8.1 & 4.8 & -13.1 \\
\hline Nonmerchant services & -2.8 & -23.3 & -3.9 & 1.0 & -8.2 \\
\hline Central government & 4.3 & 0.8 & -7.6 & -2.0 & -7.2 \\
\hline Technical assistance & -15.8 & -78.1 & 34.8 & 22.9 & -13.5 \\
\hline GDP at factor costs & -1.2 & -6.2 & 1.1 & 2.0 & 3.6 \\
\hline Indirect taxes & 13.6 & -37.6 & 10.8 & 10.6 & 3.8 \\
\hline Customs duties & 0.5 & -2.9 & -4.7 & -4.3 & 61.4 \\
\hline GDP at market prices & -0.6 & -7.6 & 1.3 & 2.2 & 4.1 \\
\hline
\end{tabular}

Sources: C.A.R. authorities; and Fund staff estimates.

'Since the base year (1985) is outdated and being updated, the C.A.R. authorities compute real GDP growth by comparing output at previous year prices (Table 1) with previous year's output at current prices. 
Table 4. Central African Republic: Supply and Use of Resources at Current Prices, 2002-06

\begin{tabular}{|c|c|c|c|c|c|}
\hline & 2002 & 2003 & 2004 & 2005 & 2006 \\
\hline & \multicolumn{5}{|c|}{ (In billions of CFA francs) } \\
\hline Aggregate supply & 872.5 & 814.0 & 820.8 & 875.3 & 950.2 \\
\hline GDP & 726.2 & 694.7 & 690.6 & 723.0 & 781.0 \\
\hline Imports of goods and services & 146.3 & 119.3 & 130.1 & 152.3 & 169.2 \\
\hline Aggregate demand & 872.5 & 814.0 & 820.8 & 875.4 & 950.2 \\
\hline Consumption ${ }^{1}$ & 694.8 & 682.7 & 689.8 & 721.5 & 772.8 \\
\hline Central government & 93.7 & 72.7 & 70.7 & 94.6 & 82.0 \\
\hline Private sector & 601.2 & 610.1 & 619.1 & 626.9 & 690.8 \\
\hline Gross fixed capital formation & 65.1 & 41.8 & 42.2 & 64.0 & 69.1 \\
\hline Central government & 34.5 & 14.6 & 13.7 & 28.8 & 25.6 \\
\hline Private sector & 30.5 & 27.2 & 28.6 & 35.2 & 43.5 \\
\hline Changes in stocks & 0.0 & 0.0 & 0.0 & 0.0 & 0.0 \\
\hline Exports of goods and services & 112.6 & 89.4 & 88.7 & 89.9 & 108.4 \\
\hline Resource gap & -33.6 & -29.9 & -41.4 & -62.5 & -60.8 \\
\hline Gross domestic savings & 31.4 & 11.9 & 0.8 & 1.5 & 8.2 \\
\hline Central government & 33.2 & -2.2 & 4.2 & 0.0 & 84.8 \\
\hline Private sector & -1.8 & 14.1 & -3.3 & 1.5 & -76.6 \\
\hline National savings & 54.0 & 27.4 & 30.5 & 16.6 & 48.4 \\
\hline Memorandum items: & \multicolumn{5}{|c|}{ (In percent of GDP) } \\
\hline National savings & 7.4 & 3.9 & 4.4 & 2.3 & 6.2 \\
\hline Central government & 7.3 & 1.6 & 3.7 & 1.5 & 15.5 \\
\hline Private sector & 0.2 & 2.3 & 0.7 & 0.8 & -9.3 \\
\hline Consumption ${ }^{1}$ & 95.7 & 98.3 & 99.9 & 99.8 & 99.0 \\
\hline Central government & 12.9 & 10.5 & 10.2 & 13.1 & 10.5 \\
\hline Private sector & 82.8 & 87.8 & 89.6 & 86.7 & 88.5 \\
\hline Gross investment & 9.0 & 6.0 & 6.1 & 8.8 & 8.8 \\
\hline Central government & 4.8 & 2.1 & 2.0 & 4.0 & 3.3 \\
\hline Private sector & 4.2 & 3.9 & 4.1 & 4.9 & 5.6 \\
\hline
\end{tabular}

Sources: C.A.R. authorities; and Fund staff estimates.

${ }^{1}$ Consumption figures reflect the authorities' definition of public consumption, which includes public sector wages. 
Table 5. Central African Republic: Agricultural Production, 2001/02-2005/06

\begin{tabular}{|c|c|c|c|c|c|}
\hline & $2001 / 02$ & $2002 / 03$ & $2003 / 04$ & $2004 / 05$ & $2005 / 06$ \\
\hline & \multicolumn{5}{|c|}{ (In thousands of tons) } \\
\hline \multicolumn{6}{|l|}{ Food crops } \\
\hline Cassava & 561.7 & 563.2 & 564.3 & 566.0 & 566.3 \\
\hline Groundnuts & 121.9 & 127.8 & 133.6 & 139.5 & 143.2 \\
\hline Millet and sorghum & 50.3 & 52.9 & 53.1 & 55.0 & 56.9 \\
\hline Maize & 107.0 & 113.0 & 119.0 & 127.0 & 128.0 \\
\hline Sesame & 39.4 & 41.1 & 42.8 & 44.5 & 46.2 \\
\hline Rice & 25.3 & 27.4 & 29.7 & 31.9 & 34.1 \\
\hline Gourds & 25.5 & 26.8 & 27.9 & 29.1 & 30.4 \\
\hline \multicolumn{6}{|l|}{ Export crops } \\
\hline Unginned cotton ${ }^{1}$ & 32.9 & 2.2 & 1.5 & 6.8 & 3.7 \\
\hline Merchant coffee & 6.2 & 5.7 & 2.2 & 4.7 & 2.5 \\
\hline Tobacco & 0.1 & 0.1 & 0.1 & 0.2 & 0.2 \\
\hline
\end{tabular}

Source: C.A.R. authorities.

${ }^{1}$ Cotton crop year ends October 31. 
Table 6. Central African Republic: Cotton Price Structure, 2001/02-05/06 ${ }^{1}$

\begin{tabular}{|c|c|c|c|c|c|}
\hline & $2001 / 02$ & $2002 / 03$ & $2003 / 04$ & $\begin{array}{r}2004 / 05 \\
\text { Est. }\end{array}$ & $\begin{array}{r}2005 / 06 \\
\text { Est. }\end{array}$ \\
\hline & \multicolumn{5}{|c|}{ (In CFA francs per kilogram; unless otherwise indicated) } \\
\hline Total cost (unginned cotton) & 202.0 & 181.0 & 151.1 & 162.5 & 168.5 \\
\hline Average producer price & 164.0 & 149.0 & 121.0 & 128.0 & 137.0 \\
\hline Other ${ }^{2}$ & 38.0 & 32.0 & 30.1 & 34.5 & 31.5 \\
\hline Ratio of ginned to unginned cotton (in percent) & 41.0 & 42.0 & 43.0 & 43.0 & 43.0 \\
\hline Equivalent cost (ginned cotton) & 492.7 & 431.0 & 355.5 & 382.4 & 396.5 \\
\hline Ginning and sales services & 90.0 & 100.9 & 100.0 & 100.0 & 100.0 \\
\hline Other general costs & 150.0 & 400.0 & 171.0 & 290.0 & 83.0 \\
\hline Transport and related costs at seaports & 95.0 & 117.0 & 106.0 & 106.0 & 103.0 \\
\hline Total costs, f.o.b. (ginned cotton) & 827.7 & $1,048.9$ & 732.5 & 878.4 & 682.5 \\
\hline Freight and insurance & 76.0 & 76.0 & 70.0 & 70.0 & 70.0 \\
\hline Total costs, c.i.f. (ginned cotton) & 903.7 & $1,124.9$ & 802.5 & 948.4 & 752.5 \\
\hline Other costs and charges & 20.0 & 24.2 & 24.0 & 24.0 & 24.0 \\
\hline Total costs & 923.7 & $1,149.1$ & 826.5 & 972.4 & 776.5 \\
\hline Average export price & 646.0 & 669.0 & 634.0 & 542.0 & 645.0 \\
\hline Average sales price $^{3}$ & 646.0 & 669.0 & 634.0 & 542.0 & 645.0 \\
\hline Export margin (loss - $)^{4}$ & -277.7 & -480.1 & -192.5 & -430.4 & -131.5 \\
\hline Export volumes (thousands ton) & 12.3 & 0.9 & 2.9 & 2.3 & 3.4 \\
\hline Total profit (loss -) (in billions of CFA francs) & -3.4 & -447.5 & -548.6 & -988.6 & -447.1 \\
\hline
\end{tabular}

Sources: National cotton company (SOCOCA) and from 2002/03 onward data are from SOCADETEX.

${ }^{1}$ Crop year ends October 31.

${ }^{2}$ Transport and transaction costs.

${ }^{3}$ Including domestic sales.

${ }^{4}$ Average export price less total costs. 
Table 7. Central African Republic: Production and Export Volumes of Forestry Products, 2002-06

\begin{tabular}{|c|c|c|c|c|c|}
\hline & 2002 & 2003 & 2004 & 2005 & 2006 \\
\hline & \multicolumn{5}{|c|}{ (In thousands of cubic meters) } \\
\hline Production & 762.9 & 586.2 & 584.7 & 525.2 & 710.0 \\
\hline Logs & 663.6 & 516.2 & 513.8 & 452.4 & 624.9 \\
\hline Sawn wood & 97.3 & 68.6 & 69.3 & 71.4 & 84.3 \\
\hline Plywood & 2.0 & 1.5 & 1.6 & 1.4 & 0.8 \\
\hline Exports & 396.3 & 284.8 & 219.4 & 199.6 & 263.5 \\
\hline Logs & 330.9 & 226.1 & 170.8 & 145.9 & 192.3 \\
\hline Sawn wood & 64.5 & 57.9 & 48.2 & 52.9 & 70.8 \\
\hline Plywood & 0.9 & 0.9 & 0.4 & 0.7 & 0.5 \\
\hline Domestic sales & 16.1 & 12.9 & 15.9 & 9.2 & 11.5 \\
\hline Sawn wood & 15.1 & 12.0 & 14.7 & 8.6 & 10.1 \\
\hline Plywood & 1.0 & 1.0 & 1.2 & 0.6 & 1.4 \\
\hline
\end{tabular}

Source: C.A.R. authorities. 
Table 8. Central African Republic: Manufacturing Output, 2002-06

\begin{tabular}{|c|c|c|c|c|c|}
\hline & 2002 & 2003 & 2004 & 2005 & 2006 \\
\hline & \multicolumn{5}{|c|}{ (In units indicated) } \\
\hline \multicolumn{6}{|l|}{ Food, beverages, and tobacco } \\
\hline Beer $^{1}$ & 132.2 & 122.7 & 118.7 & 118.9 & 123.1 \\
\hline Soft drinks ${ }^{1}$ & 42.4 & 38.6 & 41.4 & 46.7 & 51.8 \\
\hline Syrups ${ }^{1}$ & 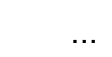 & 0.7 & 0.7 & $\ldots$ & $\ldots$ \\
\hline Cigarettes $^{2}$ & 16.6 & 15.1 & 16 & $\ldots$ & $\ldots$ \\
\hline Suger (powder) ${ }^{3}$ & 10.4 & $\ldots$ & 3.9 & $\ldots$ & $\ldots$ \\
\hline Soap $^{3}$ & 1.6 & 1.5 & 1.8 & $\ldots$ & $\ldots$ \\
\hline Palm oil ${ }^{3}$ & 2.7 & 2.2 & 2.4 & $\ldots$ & $\ldots$ \\
\hline Groundnut oil & 1.3 & $\ldots$ & $\ldots$ & $\ldots$ & $\ldots$ \\
\hline \multicolumn{6}{|l|}{ Forestry } \\
\hline Sawn wood ${ }^{4}$ & 97.3 & 68.5 & 69.3 & 71.4 & 84.3 \\
\hline \multicolumn{6}{|l|}{ Chemical products } \\
\hline Oxygen $^{5}$ & 16.2 & $\ldots$ & $\ldots$ & $\ldots$ & $\ldots$ \\
\hline Paints ${ }^{3}$ & 0.4 & $\ldots$ & $\ldots$ & $\ldots$ & $\ldots$ \\
\hline \multicolumn{6}{|l|}{ Miscellaneous } \\
\hline Aluminum household items ${ }^{3}$ & 172.4 & 86.0 & 88 & $\ldots$ & $\ldots$ \\
\hline Aluminum sheets ${ }^{3}$ & 581.6 & 249.0 & 184.1 & $\cdots$ & $\begin{array}{l}\cdots \\
\cdots\end{array}$ \\
\hline
\end{tabular}

Source: C.A.R. authorities.

${ }^{1}$ In thousands of hectoliters.

${ }^{2}$ In millions of packets.

${ }^{3}$ In thousands of tons.

${ }^{4}$ In cubic meters.

${ }^{5}$ In thousands of meters. 
Table 9. Central African Republic: Production and Export of Diamonds and Gold, 2002-06

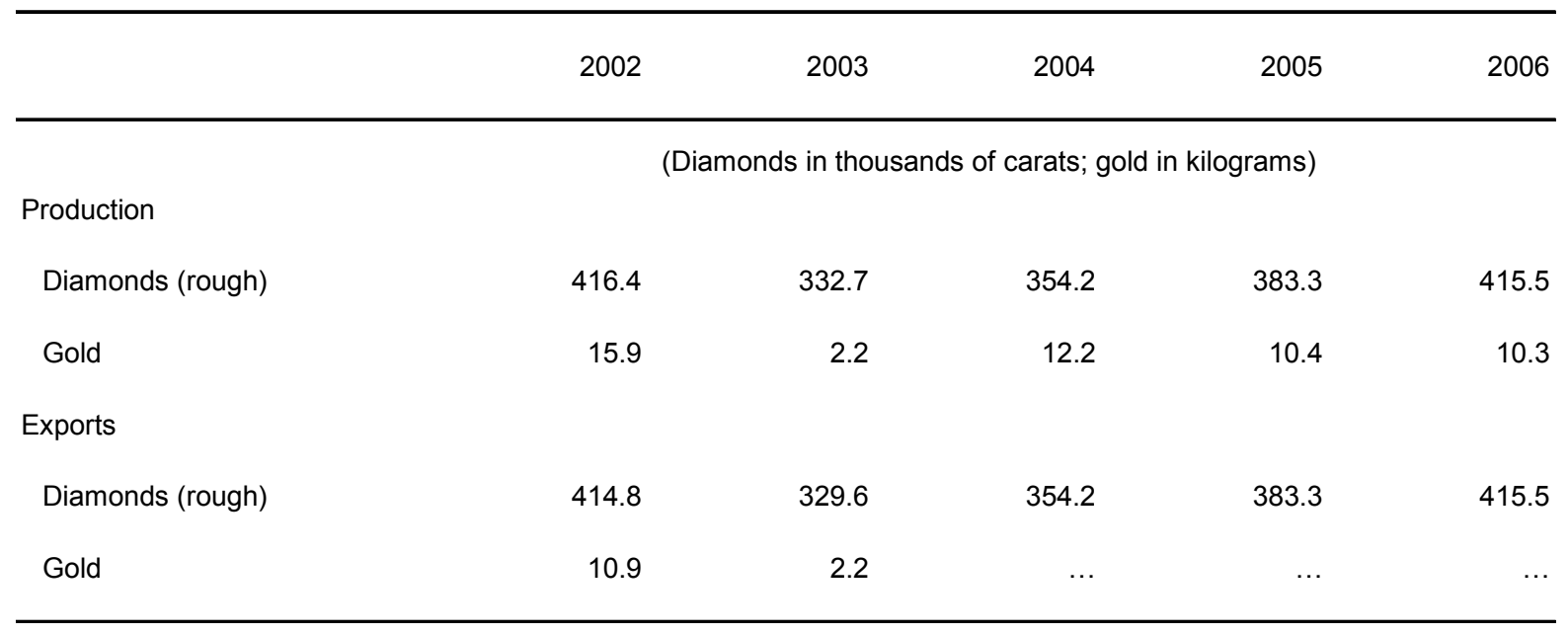

Source: C.A.R. authorities. 
Table 10. Central African Republic: Consumption of Oil Products, 2002-06

\begin{tabular}{|c|c|c|c|c|}
\hline & Premium & Oil & Diesel & \multirow{2}{*}{$\begin{array}{r}\text { Gas } \\
\text { (In tons }\end{array}$} \\
\hline & \multicolumn{3}{|c|}{ (In cubic meters) } & \\
\hline 2002 & 13,068 & 16,790 & 23,127 & 445 \\
\hline I & 3,490 & 9,475 & 6,355 & 255 \\
\hline II & 3,643 & 2,389 & 6,756 & 32 \\
\hline III & 3,318 & 2,445 & 5,809 & 136 \\
\hline IV & 2,617 & 2,481 & 4,207 & 22 \\
\hline 2003 & 9,886 & 7,594 & 15,633 & 124 \\
\hline I & 1,856 & 1,504 & 3,378 & 31 \\
\hline II & 3,056 & 2,087 & 4,572 & 34 \\
\hline III & 3,007 & 2,361 & 4,747 & 34 \\
\hline IV & 1,967 & 1,642 & 2,936 & 25 \\
\hline 2004 & 12,632 & 14,167 & 20,035 & 152 \\
\hline I & 2,981 & 3,343 & 4,332 & 37 \\
\hline II & 3,114 & 3,506 & 5,839 & 37 \\
\hline III & 3,136 & 3,617 & 4,808 & 37 \\
\hline IV & 3,402 & 3,701 & 5,057 & 42 \\
\hline 2005 & 14,698 & 16,773 & 22,579 & 165 \\
\hline 1 & 3,540 & 3,457 & 5,401 & 39 \\
\hline II & 3,563 & 4,221 & 5,602 & 41 \\
\hline III & 3,529 & 4,326 & 5,419 & 40 \\
\hline IV & 4,066 & 4,769 & 6,158 & 45 \\
\hline 2006 & 15,766 & 18,463 & 22,844 & 169 \\
\hline 1 & 3,498 & 3,561 & 5,486 & 42 \\
\hline II & 4,127 & 4,385 & 6,247 & 45 \\
\hline III & 3,916 & 4,696 & 5,322 & 42 \\
\hline IV & 4,225 & 5,821 & 5,789 & 40 \\
\hline
\end{tabular}

Source: C.A.R. authorities. 
Table 11. Central African Republic: Price Structure of Petroleum Products, 2002-06

\begin{tabular}{|c|c|c|c|c|c|c|c|c|c|c|}
\hline & \multicolumn{5}{|c|}{ Premium gasoline } & \multicolumn{5}{|c|}{ Kerosene } \\
\hline & 2002 & 2003 & 2004 & 2005 & 2006 & 2002 & 2003 & 2004 & 2005 & 2006 \\
\hline & \multicolumn{10}{|c|}{ (In CFA francs per liter) } \\
\hline $\begin{array}{l}\text { Average Platt's price over } \\
\text { the last three months }\end{array}$ & 162.4 & 148.2 & 263.4 & 341.0 & 304.7 & 164.6 & 147.6 & 363.9 & 370.8 & 347.7 \\
\hline $\begin{array}{l}\text { Transport cost from Ango-Ango } \\
\text { to Matadi }\end{array}$ & 43.3 & 43.3 & 43.3 & 43.3 & 43.3 & 43.3 & 43.3 & 43.3 & 43.3 & 43.3 \\
\hline C.i.f. price, Matadi & 205.7 & 191.5 & 306.7 & 384.4 & 348.0 & 207.9 & 190.9 & 407.2 & 414.1 & 391.0 \\
\hline Other transport costs & 43.8 & 54.7 & 59.2 & 59.2 & 58.0 & 46.1 & 58.5 & 60.0 & 60.3 & 59.0 \\
\hline Reference border prices & 249.5 & 246.2 & 365.9 & 443.6 & 406.0 & 254.0 & 249.4 & 467.2 & 474.4 & 450.0 \\
\hline Taxes and duties & 136.4 & 134.4 & 154.7 & 165.1 & 173.1 & 2.1 & 2.3 & 8.8 & 6.6 & 2.3 \\
\hline Import duties & 0.0 & 0.0 & 0.0 & 0.0 & 0.0 & 0.0 & 0.0 & 0.0 & 0.0 & 0.0 \\
\hline Complementary tax & 0.0 & 0.0 & 0.0 & 0.0 & 0.0 & 0.0 & 0.0 & 0.0 & 0.0 & 0.0 \\
\hline Specific domestic tax & 0.0 & 0.0 & 0.0 & 0.0 & 0.0 & 0.0 & 0.0 & 0.0 & 0.0 & 0.0 \\
\hline Special tax & 0.0 & 0.0 & 0.0 & 0.0 & 0.0 & 0.0 & 0.0 & 0.0 & 0.0 & 0.0 \\
\hline Road use tax & 55.0 & 55.0 & 55.0 & 55.0 & 55.0 & 0.0 & 0.0 & 0.0 & 0.0 & 0.0 \\
\hline Miscellaneous charges & 0.0 & 0.0 & 0.0 & 0.0 & 0.0 & 0.0 & 0.0 & 0.0 & 0.0 & 0.0 \\
\hline Redevance informatique & 0.4 & 0.4 & 1.0 & 1.3 & 1.1 & 0.4 & 0.4 & 1.5 & 1.5 & 1.4 \\
\hline Custom duties & 16.0 & 14.5 & 19.8 & 16.7 & 22.9 & 0.0 & 0.0 & 0.0 & 0.0 & 0.0 \\
\hline Value-added Tax & 63.4 & 63.0 & 75.2 & 87.6 & 89.8 & 0.0 & 0.0 & 0.0 & 0.0 & 0.0 \\
\hline Community intergration tax & 1.6 & 1.5 & 2.8 & 3.6 & 3.3 & 1.7 & 1.9 & 4.1 & 4.2 & 3.3 \\
\hline Price structure administration & 1.0 & 1.0 & 1.0 & 1.0 & 1.0 & 1.0 & 1.0 & 1.0 & 1.0 & 1.0 \\
\hline Garanteed price in Bangui & 386.9 & 381.5 & 520.6 & 608.8 & 579.1 & 257.0 & 252.6 & 476.0 & 481.0 & 458.0 \\
\hline Storage financing cost & 5.0 & 0.0 & 0.0 & 0.0 & 0.0 & 5.0 & 0.0 & 0.0 & 0.0 & 0.0 \\
\hline Storage fees & 15.6 & 21.5 & 21.5 & 21.5 & 21.5 & 14.3 & 20.2 & 20.2 & 21.0 & 18.0 \\
\hline Retail margin & 50.0 & 50.0 & 50.0 & 50.0 & 50.0 & 50.0 & 50.0 & 50.0 & 50.0 & 50.0 \\
\hline Wholesale margin & 12.0 & 12.0 & 12.0 & 12.0 & 12.0 & 12.0 & 12.0 & 12.0 & 12.0 & 19.0 \\
\hline Equalization fees & 0.0 & 0.0 & 0.0 & 0.0 & 0.0 & 0.0 & 0.0 & 0.0 & 0.0 & 0.0 \\
\hline Financial fees & 11.4 & 11.4 & 14.0 & 14.0 & 19.0 & 11.9 & 11.7 & 18.4 & 18.6 & 19.0 \\
\hline Transportation costs & 4.0 & 4.0 & 4.0 & 4.0 & 4.0 & 4.0 & 4.0 & 4.0 & 4.0 & 4.0 \\
\hline Research and exploitation & 0.0 & 0.0 & 0.0 & 0.0 & 0.0 & 0.0 & 0.0 & 0.0 & 0.0 & 0.0 \\
\hline Average sales price & 484.9 & 480.4 & 559.0 & 640.0 & 616.1 & 354.2 & 350.6 & 508.0 & 514.0 & 499.9 \\
\hline Unit tax on petroleum products & 165.1 & 169.7 & 91.0 & 10.0 & 83.9 & -4.2 & -0.6 & -158.0 & -164.0 & -114.9 \\
\hline Sales price at pump & 650.0 & 650.1 & 650.0 & 700.0 & 700.0 & 350.0 & 350.0 & 350.0 & 385.0 & 385.0 \\
\hline
\end{tabular}

Source: National oil import company (PETROCA). 
Table 11. Central African Republic: Price Structure of Petroleum Products, 2002-06 (concluded)

\begin{tabular}{|c|c|c|c|c|c|c|c|c|c|c|}
\hline & \multicolumn{5}{|c|}{ Diesel fuel } & \multicolumn{5}{|c|}{ Heavy fuel } \\
\hline & 2002 & 2003 & 2004 & 2005 & 2006 & 2002 & 2003 & 2004 & 2005 & 2006 \\
\hline & \multicolumn{10}{|c|}{ (In CFA francs per liter) } \\
\hline $\begin{array}{l}\text { Average Platt's price over } \\
\text { the last three months }\end{array}$ & 167.0 & 149.4 & 226.8 & 338.3 & 328.4 & 128.5 & 116.0 & 115.0 & 178.3 & 163.3 \\
\hline $\begin{array}{l}\text { Transport cost from Ango-Ango } \\
\text { to Matadi }\end{array}$ & 43.3 & 43.3 & 43.3 & 43.3 & 43.3 & 69.5 & 69.5 & 69.5 & 69.5 & 69.5 \\
\hline C.i.f. price, Matadi & 210.3 & 192.7 & 270.1 & 381.6 & 371.7 & 198.0 & 185.5 & 184.5 & 247.8 & 232.8 \\
\hline Other transport costs & 49.1 & 61.5 & 65.3 & 65.0 & 63.0 & 53.9 & 53.9 & 58.3 & 58.3 & 56.0 \\
\hline Reference border prices & 259.4 & 254.2 & 335.4 & 446.6 & 434.7 & 251.9 & 239.4 & 242.8 & 306.1 & 288.8 \\
\hline Taxes and duties & 140.1 & 137.9 & 165.0 & 183.6 & 137.9 & 0.3 & 0.3 & 3.1 & 2.6 & 1.4 \\
\hline Import duties & 0.0 & 0.0 & 0.0 & 0.0 & 0.0 & 0.0 & 0.0 & 0.0 & 0.0 & 0.0 \\
\hline Complementary tax & 0.0 & 0.0 & 0.0 & 0.0 & 0.0 & 0.0 & 0.0 & 0.0 & 0.0 & 0.0 \\
\hline Specific domestic tax & 0.0 & 0.0 & 0.0 & 0.0 & 0.0 & 0.0 & 0.0 & 0.0 & 0.0 & 0.0 \\
\hline Special tax & 0.0 & 0.0 & 0.0 & 0.0 & 0.0 & 0.0 & 0.0 & 0.0 & 0.0 & 0.0 \\
\hline Road use tax & 55.0 & 55.0 & 55.0 & 55.0 & 55.0 & 0.0 & 0.0 & 0.0 & 0.0 & 0.0 \\
\hline Miscellaneous charges & 0.0 & 0.0 & 0.0 & 0.0 & 0.0 & 0.0 & 0.0 & 0.0 & 0.0 & 0.0 \\
\hline Redevance informatique & 0.4 & 0.4 & 1.1 & 1.44 & 1.4 & 0.3 & 0.3 & 0.5 & 0.9 & 0.8 \\
\hline Custom duties & 16.9 & 15.4 & 22.7 & 28.8 & 15.4 & 0.0 & 0.0 & 0.0 & 0.0 & 0.0 \\
\hline Value-added Tax (VAT) 18 percent & 66.1 & 65.6 & 82.0 & 94.8 & 101.7 & 0.0 & 0.0 & 0.0 & 0.0 & 0.0 \\
\hline Community intergration tax & 1.7 & 1.5 & 3.2 & 4.0 & 1.5 & 0.0 & 0.0 & 1.6 & 0.7 & 0.6 \\
\hline Price structure administration & 1.0 & 1.0 & 1.0 & 1.0 & 1.0 & 1.0 & 1.0 & 1.0 & 1.0 & 1.0 \\
\hline Garanteed price in Bangui & 400.5 & 393.1 & 500.4 & 630.2 & 572.6 & 253.3 & 240.7 & 245.9 & 308.7 & 291.2 \\
\hline Storage financing cost & 5.0 & 0.0 & 0.0 & 0.0 & 0.0 & 5.1 & 0.0 & 0.0 & 0.0 & 0.0 \\
\hline Storage fees & 14.3 & 20.3 & 20.3 & 20.3 & 18.3 & 14.3 & 20.1 & 20.1 & 20.1 & 20.1 \\
\hline Retail margin & 50.0 & 50.0 & 50.0 & 50.0 & 50.0 & 50.0 & 50.0 & 50.0 & 50.0 & 50.0 \\
\hline Wholesale margin & 12.0 & 12.0 & 12.0 & 12.0 & 12.0 & 0.0 & 0.0 & 0.0 & 0.0 & 0.0 \\
\hline Equalization fees & 0.0 & 0.0 & 0.0 & 0.0 & 0.0 & 0.0 & 0.0 & 0.0 & 0.0 & 0.0 \\
\hline Financial fees & 12.1 & 12.0 & 15.6 & 12.0 & 16.0 & 11.8 & 10.9 & 11.3 & 14.2 & 13.4 \\
\hline Transportation costs & 4.0 & 4.0 & 4.0 & 4.0 & 4.0 & 4.0 & 4.0 & 4.0 & 4.0 & 4.0 \\
\hline Research and exploitation & 0.0 & 0.0 & 0.0 & 0.0 & 0.0 & 0.0 & 0.0 & 0.0 & 0.0 & 0.0 \\
\hline Average sales price & 498.9 & 492.4 & 603.0 & 688.0 & 690.9 & 339.4 & 326.7 & 331.0 & 398.0 & 378.7 \\
\hline Unit tax on petroleum products & 76.1 & 82.6 & -28.0 & -113.0 & -15.9 & 50.6 & 63.3 & 59.0 & 59.0 & 30.4 \\
\hline Sales price at pump & 575.0 & 575.0 & 575.0 & 575.0 & 675.0 & 390.0 & 390.0 & 390.0 & 390.0 & 410.0 \\
\hline
\end{tabular}

Source: National oil import company (PETROCA). 
Table 12. Central African Republic: Consumer Price Index, 2002-06

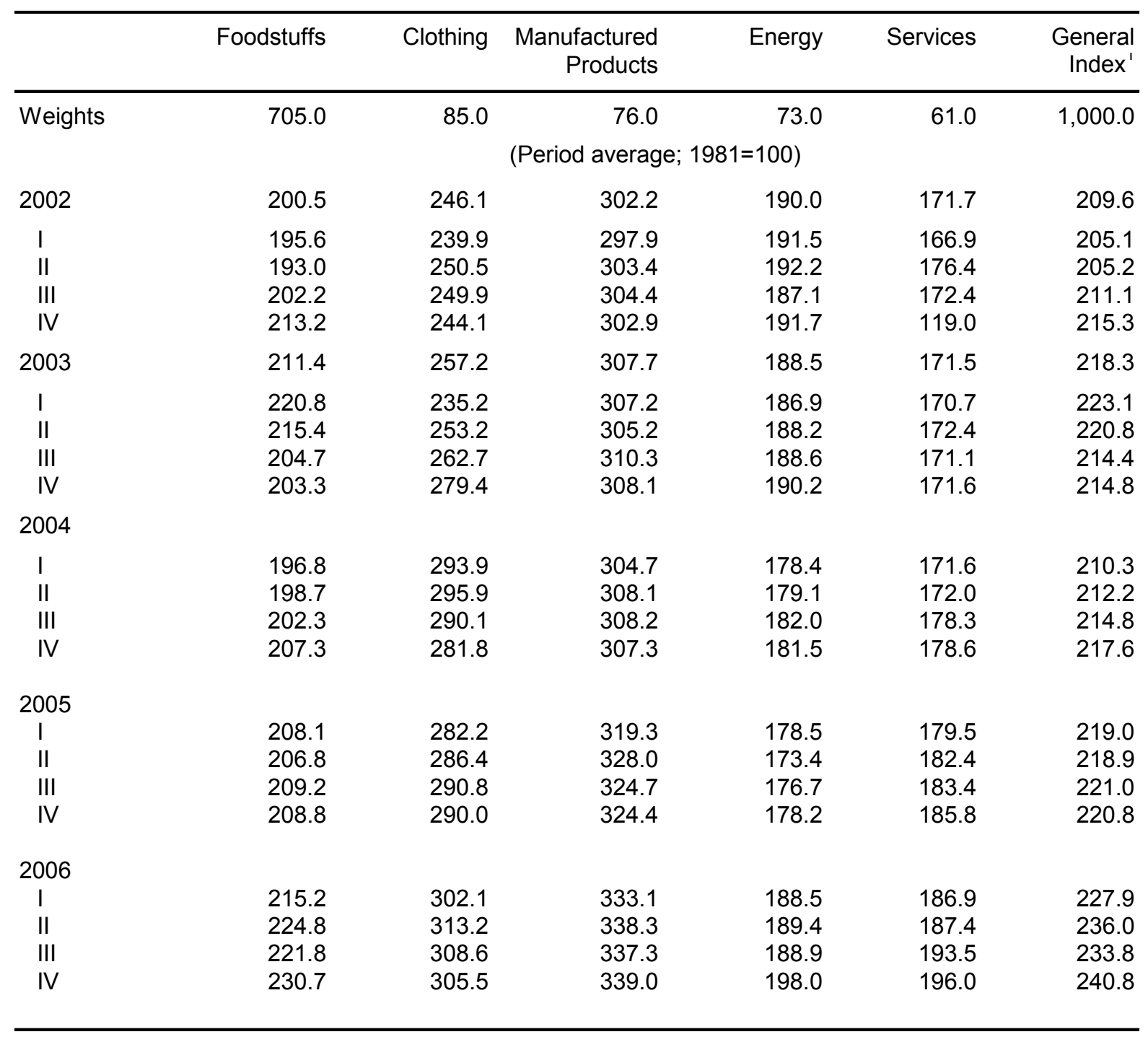

Source: C.A.R. authorities.

'Based on consumption basket for nationals living in Bangui. Averages are computed on the basis of weighted sectoral averages for the given time periods. 
Table 13. Central African Republic: Wholesale Price Index, 2002-06

\begin{tabular}{|c|c|c|c|c|c|}
\hline & Foodstuffs & Clothing & $\begin{array}{r}\text { Manufactured } \\
\text { Products }\end{array}$ & Energy & $\begin{array}{l}\text { General } \\
\text { Index }\end{array}$ \\
\hline \multirow[t]{2}{*}{ Weights } & 424.0 & 83.0 & 262.0 & 231.0 & $1,000.0$ \\
\hline & \multicolumn{5}{|c|}{ (Period average; $1981=100$ ) } \\
\hline 2002 & 298.6 & 466.3 & 265.4 & 307.3 & 268.1 \\
\hline 1 & 242.4 & 408.7 & 250.2 & 306.0 & 273.0 \\
\hline II & 392.3 & 460.2 & 261.3 & 307.5 & 344.0 \\
\hline III & 278.3 & 504.3 & 280.7 & 307.5 & 304.4 \\
\hline IV & 281.3 & 491.9 & 269.4 & 308.1 & 301.9 \\
\hline 2003 & 278.2 & 506.7 & 273.3 & 309.1 & 268.1 \\
\hline I & 286.4 & 492.0 & 273.3 & 309.1 & 305.3 \\
\hline II & 276.4 & 540.0 & 273.0 & 309.1 & 304.9 \\
\hline III & 274.0 & 524.6 & 276.7 & 309.1 & 303.6 \\
\hline IV & 276.0 & 470.3 & 270.3 & 309.1 & 298.3 \\
\hline \multicolumn{6}{|l|}{2004} \\
\hline I & 268.60 & 490.58 & 268.55 & 309.13 & 296.4 \\
\hline II & 270.9 & 522.2 & 275.3 & 309.1 & 301.7 \\
\hline III & 276.7 & 522.4 & 273.6 & 309.1 & 303.8 \\
\hline IV & 276.4 & 522.3 & 274.2 & 308.6 & 303.7 \\
\hline \multicolumn{6}{|l|}{2005} \\
\hline 1 & 287.1 & 478.3 & 271.3 & 307.5 & 303.6 \\
\hline II & 296.2 & 449.0 & 272.3 & 307.5 & 305.3 \\
\hline III & 294.2 & 445.3 & 266.1 & 307.5 & 302.5 \\
\hline IV & 338.5 & 448.9 & 264.9 & 307.5 & 321.2 \\
\hline \multicolumn{6}{|l|}{2006} \\
\hline 1 & 343.7 & 457.0 & 266.9 & 340.9 & 332.3 \\
\hline II & 338.6 & 459.5 & 278.5 & 340.9 & 333.4 \\
\hline III & 346.3 & 473.3 & 280.9 & 340.9 & 338.5 \\
\hline IV & 342.7 & 506.1 & 276.8 & 340.9 & 338.6 \\
\hline
\end{tabular}

Source: C.A.R. authorities.

'Based on consumption basket for nationals living in Bangui. Averages are computed on the basis of weighted sectoral averages for the given time periods. 
Table 14. Central African Republic: Sectoral Composition of the Public Investment Program, 2002-06 ${ }^{1}$

\begin{tabular}{|c|c|c|c|c|c|}
\hline & 2002 & 2003 & 2004 & 2005 & 2006 \\
\hline \multicolumn{6}{|c|}{ (In billions of CFA francs) } \\
\hline Rural development & 6.3 & 3.1 & $\ldots$ & $\ldots$ & $\ldots$ \\
\hline Agriculture & 3.5 & 0.5 & $\ldots$ & $\ldots$ & $\ldots$ \\
\hline Livestock & 0.4 & 0.2 & $\ldots$ & $\ldots$ & $\ldots$ \\
\hline Forestry & 2.1 & 1.7 & $\ldots$ & $\ldots$ & $\ldots$ \\
\hline Fisheries & 0.0 & 0.0 & $\ldots$ & $\ldots$ & $\ldots$ \\
\hline Hunting and fishing & 0.3 & 0.7 & $\ldots$ & $\ldots$ & $\ldots$ \\
\hline Industry and mining & 0.0 & 0.0 & $\ldots$ & $\ldots$ & $\ldots$ \\
\hline Industry & 0.0 & 0.0 & $\ldots$ & $\ldots$ & $\ldots$ \\
\hline Mining & 0.0 & 0.0 & $\ldots$ & $\ldots$ & $\ldots$ \\
\hline Economic infrastructure & 20.0 & 5.9 & $\ldots$ & $\ldots$ & $\ldots$ \\
\hline Energy & 0.0 & 0.0 & $\ldots$ & $\ldots$ & $\ldots$ \\
\hline Water & 0.4 & 0.4 & $\ldots$ & $\ldots$ & $\ldots$ \\
\hline Habitat; urban development & 9.9 & 4.0 & $\ldots$ & $\ldots$ & $\ldots$ \\
\hline Infrastructure & 9.7 & 1.5 & $\ldots$ & $\ldots$ & $\ldots$ \\
\hline Land transport & 9.6 & 1.5 & $\ldots$ & $\ldots$ & $\ldots$ \\
\hline River transport & 0.0 & 0.0 & $\ldots$ & $\ldots$ & $\ldots$ \\
\hline Air transport & 0.0 & 0.0 & $\ldots$ & $\ldots$ & $\ldots$ \\
\hline Post and telecommunications & 0.0 & 0.0 & $\ldots$ & $\ldots$ & $\ldots$ \\
\hline Radio, television, and press & 0.1 & 0.2 & $\ldots$ & $\ldots$ & $\ldots$ \\
\hline \multirow[t]{2}{*}{ Commerce, insurance, and tourism } & 0.2 & 0.0 & $\ldots$ & $\ldots$ & $\ldots$ \\
\hline & & & $\ldots$ & $\ldots$ & $\ldots$ \\
\hline Social infrastructure & 16.2 & 13.2 & $\ldots$ & $\ldots$ & $\ldots$ \\
\hline Health & 5.6 & 5.5 & $\ldots$ & $\ldots$ & $\ldots$ \\
\hline Education & 1.9 & 0.5 & $\ldots$ & $\ldots$ & $\ldots$ \\
\hline Social affairs & 1.5 & 2.0 & $\ldots$ & $\ldots$ & $\ldots$ \\
\hline \multirow[t]{2}{*}{ Administration } & 7.2 & 5.2 & $\ldots$ & $\ldots$ & $\ldots$ \\
\hline & & & $\ldots$ & $\ldots$ & $\ldots$ \\
\hline \multirow[t]{2}{*}{ Unallocated } & 0.0 & 0.0 & $\ldots$ & $\ldots$ & $\ldots$ \\
\hline & & & $\cdots$ & $\cdots$ & $\cdots$ \\
\hline Total & 42.8 & 22.4 & $\ldots$ & $\ldots$ & $\ldots$ \\
\hline \multicolumn{6}{|l|}{ Sources of financing } \\
\hline Domestic $^{2}$ & 5.3 & 3.1 & 4.1 & $\ldots$ & $\ldots$ \\
\hline External & 38.0 & 20.0 & 25.5 & $\ldots$ & $\ldots$ \\
\hline Grants & 32.0 & 15.6 & 20.8 & $\ldots$ & $\ldots$ \\
\hline Loans & 6.0 & 4.4 & 4.6 & $\ldots$ & $\ldots$ \\
\hline Total & 43.3 & 23.1 & 29.6 & $\ldots$ & $\ldots$ \\
\hline
\end{tabular}

Source: C.A.R. authorities.

${ }^{1}$ Results for some years do not correspond to those in Table 15 owing to differences concerning the amount of grants effectively disbursed at year end.

${ }^{2}$ Includes counterparts of customs duties on project-related imports. 
Table 15. Central African Republic: Central Government Operations, 2002-06

\begin{tabular}{|c|c|c|c|c|c|}
\hline & 2002 & 2003 & 2004 & 2005 & 2006 \\
\hline & \multicolumn{5}{|c|}{ (In billions of CFA francs; unless otherwise indicated) } \\
\hline Revenue & 78.4 & 53.7 & 55.8 & 58.6 & 73.3 \\
\hline Tax revenue & 61.4 & 45.3 & 48.3 & 50.6 & 60.3 \\
\hline Nontax revenue & 17.0 & 8.4 & 7.5 & 8.0 & 12.9 \\
\hline Expenditure & -114.6 & -85.5 & -93.4 & -120.4 & -109.8 \\
\hline Current primary expenditure & -57.3 & -58.1 & -66.7 & -75.4 & -62.5 \\
\hline Interest due & -11.3 & -7.9 & -8.5 & -6.6 & -13.1 \\
\hline Capital expenditure & -46.0 & -19.5 & -18.2 & -38.4 & -34.1 \\
\hline Of which: domestically financed & -13.5 & -10.5 & -7.7 & -8.4 & -7.5 \\
\hline \multicolumn{6}{|l|}{ Overall balance, commitment basis } \\
\hline Excluding grants & -36.2 & -31.8 & -37.7 & -61.8 & -36.5 \\
\hline Of which: narrow primary balance ${ }^{1}$ & 7.6 & -14.9 & -18.6 & -25.3 & 3.3 \\
\hline Including grants & -8.6 & -21.8 & -15.0 & -32.4 & 55.1 \\
\hline Change in arrears (reduction -) & 21.3 & 28.4 & 21.1 & 26.9 & -50.1 \\
\hline Domestic & -4.7 & 4.1 & 2.9 & 9.4 & -9.5 \\
\hline External & 26.1 & 24.3 & 18.2 & 17.5 & -40.6 \\
\hline Overall balance, cash basis & -14.8 & -3.5 & 6.1 & -5.5 & 5.0 \\
\hline External financing, net & 14.5 & 3.5 & -14.6 & -4.5 & -12.9 \\
\hline Projects & 32.5 & 9.0 & 2.6 & 3.2 & 3.0 \\
\hline Grants & 26.5 & 6.0 & 0.0 & 0.0 & 0.0 \\
\hline Loans & 6.0 & 3.0 & 2.6 & 3.2 & 3.0 \\
\hline Program & 2.3 & 9.1 & 0.0 & 4.0 & 0.0 \\
\hline Grants & 1.0 & 4.1 & 0.0 & 0.0 & 0.0 \\
\hline Loans & 1.3 & 5.0 & 0.0 & 4.0 & 0.0 \\
\hline Amortization due & -20.4 & -21.6 & -17.2 & -11.8 & -15.9 \\
\hline External debt relief & 0.0 & 0.0 & 0.0 & 0.0 & 0.0 \\
\hline Domestic financing, net & 0.4 & 4.8 & 8.5 & 10.0 & 7.9 \\
\hline Banking system & 1.0 & 5.2 & 8.5 & 10.0 & 7.9 \\
\hline Bank of Central African States & 2.1 & 3.1 & 9.9 & 7.6 & 3.7 \\
\hline Counterpart to IMF resources & 0.0 & 0.0 & 3.1 & -2.6 & 2.2 \\
\hline Other & 2.1 & 3.1 & 0.0 & 0.0 & 0.0 \\
\hline Commercial banks & -1.1 & 2.1 & -1.4 & 2.4 & 4.2 \\
\hline Nonbank & -0.6 & -0.4 & 0.0 & 0.0 & 0.0 \\
\hline Memorandum items: & \multicolumn{5}{|c|}{ (In percent of GDP) } \\
\hline Revenue & 10.8 & 7.7 & 8.1 & 8.1 & 9.5 \\
\hline $\begin{array}{l}\text { Expenditure } \\
\text { Of which: military expenditure }\end{array}$ & -15.8 & -12.3 & -13.5 & -16.7 & -14.2 \\
\hline Current primary expenditure & -7.9 & -8.3 & -9.7 & -10.4 & -8.1 \\
\hline Interest due & -1.6 & -1.1 & -1.2 & -1.0 & -1.1 \\
\hline Capital expenditure & -6.3 & -2.8 & -2.6 & -5.3 & -4.9 \\
\hline \multicolumn{6}{|l|}{ Balance, commitments basis (deficit -) } \\
\hline Excluding grants & -5.0 & -4.6 & -5.4 & -8.6 & -4.6 \\
\hline Including grants & -1.2 & -3.1 & -2.2 & -4.6 & 7.7 \\
\hline Narrow primary budget balance ${ }^{1}$ & 1.0 & -2.1 & -1.6 & -2.3 & 1.4 \\
\hline
\end{tabular}

Sources : C.A.R. authorities; and Fund staff estimates.

${ }^{1}$ Excludes interest payments and externally financed investment. 
Table 16. Central African Republic: Central Government Revenue, 2002-06

\begin{tabular}{|c|c|c|c|c|c|}
\hline & 2002 & 2003 & 2004 & 2005 & 2006 \\
\hline & \multicolumn{5}{|c|}{ (In billions of CFA francs) } \\
\hline Total revenue & 78.4 & 53.7 & 55.8 & 58.6 & 73.4 \\
\hline Tax revenue & 61.4 & 45.0 & 48.3 & 50.6 & 60.3 \\
\hline Taxes on income and profits & 17.2 & 13.9 & 15.6 & 14.7 & 17.5 \\
\hline Companies & 0.0 & 3.9 & 3.3 & 3.4 & 7.7 \\
\hline Individuals & 0.0 & 4.5 & 5.6 & 4.3 & 5.2 \\
\hline Other & 17.2 & 5.6 & 6.6 & 7.1 & 4.6 \\
\hline Taxes on goods and services & 33.3 & 20.7 & 22.1 & 26.4 & 27.7 \\
\hline Turnover tax & 0.0 & 9.8 & 12.1 & 15.2 & 17.2 \\
\hline Excise tax & 0.0 & 1.4 & 1.1 & 0.7 & 1.4 \\
\hline Other & 33.3 & 9.6 & 9.1 & 10.5 & 9.1 \\
\hline Taxes on international trade & 10.9 & 10.4 & 10.6 & 9.5 & 15.2 \\
\hline Import taxes and duties & 7.6 & 7.3 & 7.2 & 7.0 & 11.8 \\
\hline Customs duties & 0.0 & 7.3 & 7.2 & 7.0 & 11.8 \\
\hline Other taxes and duties & 7.6 & 0.0 & 0.0 & 0.0 & 0.0 \\
\hline Export taxes and duties & 3.2 & 3.1 & 3.5 & 2.5 & 3.5 \\
\hline Nontax revenue & 17.0 & 8.6 & 7.5 & 8.0 & 12.9 \\
\hline Dividends & 0.0 & 0.6 & 0.2 & 0.1 & 3.2 \\
\hline \multirow[t]{2}{*}{ Other } & 17.0 & 8.1 & 7.3 & 7.8 & 9.7 \\
\hline & \multicolumn{5}{|c|}{ (In percent of GDP) } \\
\hline Total revenue & 10.8 & 7.7 & 8.1 & 8.1 & 9.5 \\
\hline Tax revenue & 8.5 & 6.5 & 7.0 & 7.0 & 7.8 \\
\hline Taxes on income and profits & 2.4 & 2.0 & 2.3 & 2.0 & 2.2 \\
\hline Taxes on goods and services & 4.6 & 3.0 & 3.2 & 3.6 & 3.6 \\
\hline Taxes on international trade & 1.5 & 1.5 & 1.5 & 1.3 & 1.9 \\
\hline Nontax revenue & 2.3 & 1.2 & 1.1 & 1.1 & 1.7 \\
\hline
\end{tabular}

Sources : C.A.R. authorities; and Fund staff estimates. 
Table 17. Central African Republic: Central Government Expenditure, 2002-06

\begin{tabular}{|c|c|c|c|c|c|}
\hline & 2002 & 2003 & 2004 & 2005 & 2006 \\
\hline & \multicolumn{5}{|c|}{ (In billions of CFA francs) } \\
\hline Total expenditure & 114.6 & 85.5 & 93.4 & 120.9 & 108.8 \\
\hline Current expenditure & 68.6 & 66.0 & 75.2 & 82.6 & 71.1 \\
\hline Current primary expenditure & 57.3 & 58.1 & 66.7 & 75.4 & 62.5 \\
\hline Wages and salaries & 33.1 & 34.5 & 38.9 & 39.2 & 37.2 \\
\hline Transfers and subsidies & 7.1 & 7.3 & 10.0 & 14.0 & 10.2 \\
\hline Goods and services & 17.1 & 16.3 & 17.8 & 22.2 & 15.2 \\
\hline Interest due & 11.3 & 7.9 & 8.5 & 7.2 & 8.6 \\
\hline External & 7.3 & 5.0 & 3.0 & 3.0 & 2.7 \\
\hline Domestic & 3.9 & 2.9 & 5.5 & 4.2 & 5.9 \\
\hline Public investment program & 46.0 & 19.5 & 18.2 & 38.4 & 34.1 \\
\hline Domestically financed & 13.5 & 10.5 & 7.7 & 8.4 & 7.5 \\
\hline \multirow[t]{2}{*}{ Externally financed } & 32.5 & 9.0 & 10.5 & 29.9 & 26.6 \\
\hline & \multicolumn{5}{|c|}{ (In percent of GDP) } \\
\hline Total expenditure & 15.8 & 12.3 & 13.5 & 16.7 & 14.1 \\
\hline Current expenditure & 9.4 & 9.5 & 10.9 & 11.4 & 9.2 \\
\hline Current primary expenditure & 7.9 & 8.3 & 9.7 & 10.4 & 8.0 \\
\hline Wages and salaries & 4.6 & 5.0 & 5.6 & 5.4 & 4.8 \\
\hline Transfers and subsidies & 1.0 & 1.0 & 1.4 & 1.9 & 1.3 \\
\hline Goods and services & 2.4 & 2.3 & 2.6 & 3.1 & 2.0 \\
\hline Interest due & 1.6 & 1.1 & 1.2 & 0.9 & 1.7 \\
\hline Public investment program & 6.3 & 2.8 & 2.6 & 5.3 & 4.4 \\
\hline Of which: domestically financed & 1.9 & 1.5 & 1.1 & 1.2 & 1.0 \\
\hline
\end{tabular}

Sources: C.A.R. authorities; and Fund staff estimates. 
21

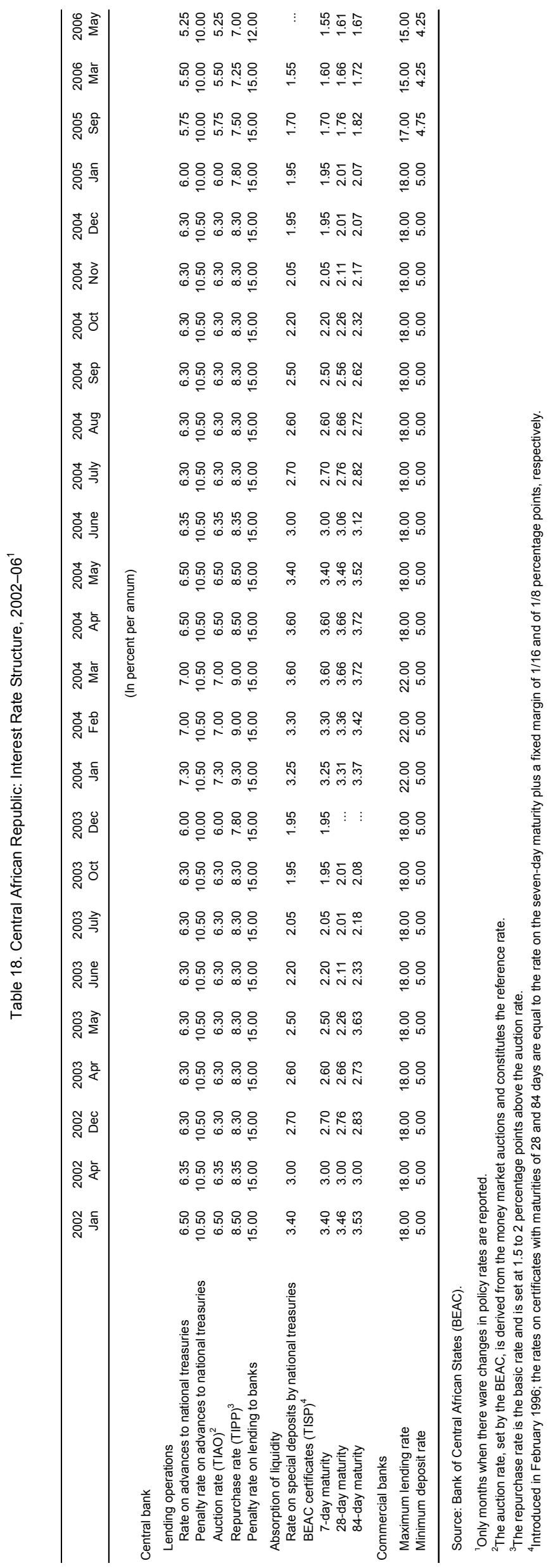


Table 19. Central African Republic: Monetary Survey, 2002-06

\begin{tabular}{|c|c|c|c|c|c|}
\hline & 2002 & 2003 & 2004 & 2005 & 2006 \\
\hline & \multicolumn{5}{|c|}{ (End of period, in billions of CFA francs) } \\
\hline Net foreign assets & 55.7 & 48.7 & 49.5 & 56.2 & 43.0 \\
\hline Central Bank & 58.1 & 52.0 & 51.4 & 59.7 & 43.6 \\
\hline Operations account & 76.9 & 68.7 & 69.6 & 77.0 & 61.6 \\
\hline Use of IMF credit & -20.9 & -19.7 & -21.2 & -20.0 & -21.0 \\
\hline Other & 2.1 & 3.0 & 3.0 & 2.6 & 2.9 \\
\hline Commercial banks & -2.4 & -3.3 & -1.9 & -3.5 & -0.6 \\
\hline Net domestic assets & 49.2 & 51.2 & 60.7 & 72.2 & 80.0 \\
\hline Domestic credit & 99.6 & 103.2 & 115.4 & 126.1 & 135.7 \\
\hline Net credit to the government & 50.2 & 55.2 & 60.2 & 72.0 & 79.3 \\
\hline Central government & 54.3 & 58.4 & 62.9 & 74.2 & 80.9 \\
\hline Other public agencies & -4.1 & -3.2 & -2.7 & -2.2 & -1.6 \\
\hline Credit to the economy & 49.4 & 48.0 & 55.2 & 54.1 & 56.4 \\
\hline Public enterprises & 8.1 & 8.9 & 6.7 & 5.6 & 5.2 \\
\hline Private sector & 41.3 & 39.1 & 48.4 & 48.5 & 51.3 \\
\hline Other items (net) & -50.3 & -52.0 & -54.6 & -53.9 & -55.7 \\
\hline Broad money & 104.9 & 99.9 & 110.2 & 128.4 & 123.0 \\
\hline Currency outside banks & 77.4 & 71.4 & 81.3 & 89.9 & 80.9 \\
\hline Deposits & 27.5 & 28.5 & 28.9 & 38.6 & 42.0 \\
\hline Demand deposits & 17.3 & 17.6 & 16.4 & 23.8 & 24.5 \\
\hline Time deposits & 10.2 & 10.9 & 12.5 & 14.7 & 17.5 \\
\hline \multicolumn{6}{|l|}{ Memorandum item: } \\
\hline \multirow[t]{2}{*}{ Velocity of broad money ${ }^{1}$} & 6.6 & 6.9 & 6.3 & 5.6 & 6.4 \\
\hline & \multicolumn{5}{|c|}{$\begin{array}{c}\text { (Annual changes in percent of broad money } \\
\text { beginning-period) }\end{array}$} \\
\hline Net foreign assets & -7.3 & -6.7 & 2.2 & 6.1 & -10.3 \\
\hline Net domestic assets & 2.9 & 1.9 & 12.0 & 10.4 & 6.0 \\
\hline Domestic credit & 2.2 & 3.4 & 13.6 & 9.8 & 7.5 \\
\hline Credit to the government (net) & -0.9 & 3.9 & 7.9 & 10.3 & 5.2 \\
\hline Credit to the economy & 6.3 & -1.3 & 6.0 & -1.0 & 1.8 \\
\hline \multirow[t]{2}{*}{ Money supply (M2) } & -4.3 & -4.8 & 14.2 & 16.5 & -4.2 \\
\hline & \multicolumn{5}{|c|}{ (Annual percentage changes) } \\
\hline Monetary base & -6.0 & -7.6 & 15.3 & 17.4 & -14.8 \\
\hline Credit to the economy & 16.3 & -2.8 & 11.6 & -1.9 & 4.3 \\
\hline Nominal GDP & 2.4 & -4.1 & -0.6 & 4.7 & 7.7 \\
\hline
\end{tabular}

Sources: Bank of Central African States (BEAC); and Fund staff estimates.

'GDP over five-quarter average of broad money. 
Table 20. Central African Republic: Central Bank Accounts, 2002-06

\begin{tabular}{|c|c|c|c|c|c|}
\hline & 2002 & 2003 & 2004 & 2005 & 2006 \\
\hline & \multicolumn{5}{|c|}{ (End of period, in billions of CFA francs) } \\
\hline Total assets & 138.3 & 129.6 & 142.7 & 164.4 & 145.9 \\
\hline Foreign assets & 79.4 & 72.0 & 73.2 & 80.6 & 65.6 \\
\hline Gold & 2.4 & 2.4 & 2.4 & 3.2 & 3.5 \\
\hline SDRs & 0.0 & 0.1 & 1.2 & 0.1 & 0.4 \\
\hline CFA franc zone currency & 0.1 & 0.8 & 0.5 & 0.2 & 0.4 \\
\hline Operations account (credit balance) & 76.9 & 68.7 & 69.6 & 77.0 & 61.6 \\
\hline Other assets & 0.0 & 0.0 & 0.1 & 0.1 & 0.0 \\
\hline Credit to the government & 49.6 & 51.3 & 60.9 & 80.6 & 75.6 \\
\hline Current accounts (Article 21) & 12.6 & 15.5 & 15.5 & 15.5 & 17.0 \\
\hline Use of IMF credit & 20.9 & 19.7 & 21.2 & 20.0 & 21.0 \\
\hline Stand-By Arrangements & 0.0 & 0.0 & 0.0 & 0.0 & 0.0 \\
\hline SAF arrangements & 0.0 & 0.0 & 0.0 & 0.0 & 0.0 \\
\hline ESAF/PRGF arrangements & 20.9 & 19.7 & 21.2 & 20.0 & 21.0 \\
\hline Consolidated loans & 14.6 & 14.6 & 14.6 & 17.2 & 18.4 \\
\hline Other $^{1}$ & 1.5 & 1.5 & & & \\
\hline Credit to deposit money banks & 3.3 & 1.8 & 4.1 & 0.0 & 0.0 \\
\hline Other assets & 6.0 & 4.5 & 4.5 & 3.2 & 4.7 \\
\hline Total liabilities & 138.3 & 129.6 & 142.7 & 164.4 & 145.9 \\
\hline Monetary base & 79.0 & 73.0 & 83.7 & 98.3 & 83.7 \\
\hline Currency in circulation & 77.4 & 71.4 & 81.3 & 89.9 & 80.9 \\
\hline Currency issued & 78.5 & 72.2 & 81.8 & 95.9 & 81.9 \\
\hline Treasury cash & -0.1 & -0.1 & -0.1 & -0.1 & -0.1 \\
\hline Currency in banks & -0.9 & -0.7 & -1.8 & -2.2 & -1.6 \\
\hline Reserves & 1.5 & 1.6 & 2.4 & 8.4 & 2.8 \\
\hline Currency in banks & 0.9 & 0.7 & 1.8 & 2.2 & 1.6 \\
\hline Bank deposits with BEAC & 0.6 & 0.9 & 0.6 & 6.1 & 1.2 \\
\hline Foreign liabilities & 21.3 & 20.0 & 22.4 & 20.9 & 22.4 \\
\hline Current accounts of foreign institutions & 10.7 & 10.2 & 9.9 & 10.0 & 10.2 \\
\hline Deposits of regional institutions & 0.1 & 0.1 & 0.5 & 6.1 & 1.2 \\
\hline Use of IMF credit & 20.9 & 19.7 & 21.2 & 20.0 & 21.0 \\
\hline Other liabilities & -10.3 & -10.0 & -9.2 & -15.2 & -10.0 \\
\hline Central government deposits & 0.8 & 0.6 & 2.2 & 1.6 & 5.4 \\
\hline Other liabilities & 35.6 & 33.9 & 33.2 & 30.7 & 33.3 \\
\hline
\end{tabular}

Source: Bank of Central African States (BEAC).

${ }^{1}$ Including unpaid interest. 
Table 21. Central African Republic: Balance Sheet of Commercial Banks, 2002-06

\begin{tabular}{lrrrrr}
\hline & 2002 & 2003 & 2004 & 2005 & 2006 \\
\hline & \multicolumn{3}{c}{ (End of perild, in billions of CFA francs) } \\
Total assets & 65.5 & 64.0 & 62.9 & 69.2 & 76.0 \\
Reserves & 1.5 & 1.6 & 2.4 & 8.4 & 2.8 \\
Foreign assets & 3.7 & 3.9 & 6.2 & 4.9 & 9.9 \\
Credit to the central government & 6.4 & 8.2 & 5.0 & 7.2 & 12.4 \\
Credit to other public agencies & 2.0 & 1.8 & 1.9 & 2.1 & 2.0 \\
Credit to the economy & 49.4 & 48.0 & 55.2 & 54.1 & 56.4 \\
$\quad$ Public enterprises & 8.1 & 8.9 & 6.7 & 5.6 & 5.2 \\
$\quad$ Private sector & 41.3 & 39.1 & 48.4 & 48.5 & 51.3 \\
Other assets & 2.6 & 0.5 & -7.7 & -7.5 & -7.4 \\
Total liabilities & 65.5 & 64.0 & 62.9 & 69.2 & 76.0 \\
Deposits & 34.4 & 34.0 & 34.3 & 43.5 & 47.2 \\
Demand deposits & 17.3 & 17.6 & 16.4 & 23.8 & 24.5 \\
Term and savings deposits & 10.2 & 10.9 & 12.5 & 14.7 & 17.5 \\
Government deposits & 0.7 & 0.5 & 0.8 & 0.7 & 1.6 \\
Deposits of other public institutions & 6.1 & 5.0 & 4.6 & 4.2 & 3.6 \\
Foreign liabilities & 6.0 & 7.2 & 8.1 & 8.4 & 10.4 \\
Short-term & 5.8 & 5.5 & 4.3 & 5.0 & 6.9 \\
$\quad$ Of which: postal debt & 1.3 & 1.3 & $\ldots$. & $\ldots$ & $\ldots$ \\
Medium- and long-term & 0.2 & 1.7 & 3.8 & 3.4 & 3.5 \\
Credit from the BEAC & 3.3 & 1.8 & 4.1 & 0.0 & 0.0 \\
Capital and reserves & 22.4 & 21.2 & 23.2 & 25.5 & 27.0 \\
Other liabilities & -0.6 & -0.2 & -6.8 & -8.2 & -8.7 \\
\hline
\end{tabular}

Source: Bank of Central African States (BEAC). 
Table 22. Central African Republic: Balance of Payments, 2002-06

\begin{tabular}{|c|c|c|c|c|c|}
\hline & 2002 & 2003 & 2004 & 2005 & $\begin{array}{r}2006 \\
\text { Est. }\end{array}$ \\
\hline & \multicolumn{5}{|c|}{ (In millions of SDRs) } \\
\hline Current account & -12.3 & -17.8 & -15.0 & -61.0 & -26.9 \\
\hline Balance of goods & 20.6 & 7.5 & -14.5 & -29.5 & -31.0 \\
\hline Exports, f.o.b. & 113.7 & 91.6 & 85.4 & 86.9 & 107.2 \\
\hline Diamonds & 40.4 & 35.2 & 35.5 & 42.3 & 42.5 \\
\hline Wood products & 57.8 & 42.5 & 36.8 & 33.3 & 51.9 \\
\hline Cotton & 7.1 & 0.7 & 2.3 & 1.4 & 0.9 \\
\hline Coffee & 1.1 & 0.9 & 1.3 & 1.2 & 1.0 \\
\hline Other & 7.4 & 12.2 & 9.5 & 8.7 & 10.8 \\
\hline Imports, f.o.b. & -93.1 & -84.1 & -99.9 & -116.4 & -138.2 \\
\hline Petroleum products & -11.2 & -9.5 & -22.8 & -22.8 & -33.9 \\
\hline Public investment program & -14.4 & -6.3 & -5.0 & -14.4 & -18.1 \\
\hline Other & -67.5 & -68.3 & -72.1 & -79.1 & -86.3 \\
\hline Services (net) & -58.0 & -44.3 & -38.5 & -51.0 & -48.1 \\
\hline Credit & 11.4 & 18.5 & 28.2 & 28.9 & 33.8 \\
\hline Debit & -69.5 & -62.7 & -66.6 & -79.9 & -82.0 \\
\hline Income & -6.7 & -1.7 & -1.1 & -1.4 & -2.7 \\
\hline Credit & 4.1 & 5.0 & 6.5 & 6.7 & 7.2 \\
\hline Debit & -10.8 & -6.7 & -7.6 & -8.1 & -9.8 \\
\hline Current transfers (net) & 31.8 & 20.7 & 39.0 & 20.9 & 54.9 \\
\hline Capital account & 15.1 & 8.5 & 23.7 & 19.9 & 106.8 \\
\hline Project grants & 14.0 & 3.5 & 4.8 & 16.4 & 18.5 \\
\hline Program grants & 1.1 & 5.0 & 18.9 & 3.5 & 88.4 \\
\hline Financial account & -53.3 & -30.6 & -33.7 & 30.9 & -49.7 \\
\hline Public sector (net) & -14.5 & -14.0 & -18.7 & -5.9 & -12.9 \\
\hline Project disbursements & 6.7 & 3.7 & 3.4 & 4.1 & 3.9 \\
\hline Program disbursements & 1.5 & 6.2 & 0.0 & 5.2 & 0.0 \\
\hline Scheduled amortization & -22.7 & -23.9 & -22.0 & -15.2 & -20.7 \\
\hline Private sector (net) & -38.8 & -16.6 & -15.0 & 36.8 & -32.9 \\
\hline Errors and omissions & 10.2 & 0.0 & 0.0 & 0.0 & 0.0 \\
\hline Overall balance & -40.4 & -39.9 & -25.0 & -10.2 & 30.2 \\
\hline Net official reserves movements ${ }^{1}$ & 11.4 & 10.0 & 1.7 & -12.4 & 22.6 \\
\hline Net Fund credit & 0.0 & 0.0 & 4.0 & -3.3 & 2.9 \\
\hline Purchases and loans & 0.0 & 0.0 & 5.7 & 0.0 & 7.0 \\
\hline Repayments (cash basis) & 0.0 & 0.0 & -1.6 & -3.3 & -4.1 \\
\hline Other reserves (increase -) & 11.4 & 10.0 & -2.3 & -9.1 & 19.7 \\
\hline Exceptional financing & 29.0 & 29.8 & 23.3 & 22.6 & -52.7 \\
\hline Debt rescheduling & 0.0 & 0.0 & 0.0 & 0.0 & 0.0 \\
\hline Debt-payments arrears (reduction -) & 29.0 & 29.8 & 23.3 & 22.6 & -52.7 \\
\hline
\end{tabular}

Sources: C.A.R. authorities; Bank of Central African States (BEAC); and Fund staff estimates. 
Table 23. Central African Republic: Balance of Payments, 2002-06 ${ }^{1}$

\begin{tabular}{|c|c|c|c|c|c|}
\hline & 2002 & 2003 & 2004 & 2005 & $\begin{array}{r}2006 \\
\text { Est. }\end{array}$ \\
\hline & \multicolumn{5}{|c|}{ (In billions of CFA francs, unless otherwise indicated ) } \\
\hline Current account & -11.1 & -14.5 & -11.8 & -47.3 & -20.7 \\
\hline Balance of goods & 18.6 & 6.1 & -11.3 & -22.9 & -23.8 \\
\hline Exports, f.o.b. & 102.3 & 74.4 & 66.7 & 67.5 & 82.4 \\
\hline Diamonds & 36.3 & 28.6 & 27.8 & 32.8 & 32.7 \\
\hline Wood products & 52.0 & 34.6 & 28.8 & 25.8 & 39.9 \\
\hline Cotton & 6.4 & 0.6 & 1.8 & 1.1 & 0.7 \\
\hline Coffee & 1.0 & 0.8 & 1.0 & 0.9 & 0.8 \\
\hline Other & 6.6 & 9.9 & 7.4 & 6.8 & 8.3 \\
\hline Imports, f.o.b. & -83.8 & -68.3 & -78.1 & -90.3 & -106.2 \\
\hline Petroleum products & -10.1 & -7.7 & -17.8 & -17.7 & -26.0 \\
\hline Public investment program & -13.0 & -5.1 & -3.9 & -11.2 & -13.9 \\
\hline Other & -60.7 & -55.5 & -56.3 & -61.4 & -66.3 \\
\hline Services (net) & -52.2 & -36.0 & -30.1 & -39.6 & -37.0 \\
\hline Credit & 10.3 & 15.0 & 22.0 & 22.4 & 26.0 \\
\hline Debit & -62.5 & -51.0 & -52.1 & -62.0 & -63.0 \\
\hline Income & -6.1 & -1.4 & -0.9 & -1.1 & -2.1 \\
\hline Credit & 3.7 & 4.1 & 5.1 & 5.2 & 5.5 \\
\hline Debit & -9.8 & -5.5 & -6.0 & -6.3 & -7.6 \\
\hline Current transfers (net) & 28.6 & 16.8 & 30.5 & 16.2 & 42.2 \\
\hline Capital account & 13.6 & 6.9 & 18.5 & 15.4 & 82.1 \\
\hline Project grants & 12.6 & 2.8 & 3.8 & 12.7 & 14.2 \\
\hline Program grants & 1.0 & 4.1 & 14.8 & 2.7 & 67.9 \\
\hline Financial account & -48.0 & -24.9 & -26.3 & 24.0 & -38.2 \\
\hline Public sector (net) & -13.1 & -11.4 & -14.6 & -4.5 & -9.9 \\
\hline Project disbursements & 6.0 & 3.0 & 2.6 & 3.2 & 3.0 \\
\hline Program disbursements & 1.3 & 5.0 & 0.0 & 4.0 & 0.0 \\
\hline Scheduled amortization & -20.4 & -19.4 & -17.2 & -11.8 & -15.9 \\
\hline Private sector (net) & -34.9 & -13.5 & -11.7 & 28.6 & -25.3 \\
\hline Errors and omissions & 9.2 & 0.0 & 0.0 & 0.0 & 0.0 \\
\hline Overall balance & -36.3 & -32.4 & -19.5 & -7.9 & 23.2 \\
\hline Net official reserves movements ${ }^{1}$ & 10.3 & 8.1 & 1.4 & -9.6 & 17.3 \\
\hline Net Fund credit & 0.0 & 0.0 & 3.1 & -2.6 & 2.2 \\
\hline Purchases and loans & 0.0 & 0.0 & 4.4 & 0.0 & 5.4 \\
\hline Repayments (cash basis) & 0.0 & 0.0 & -1.3 & -2.6 & -3.1 \\
\hline Other reserves (increase -) & 10.3 & 8.1 & -1.8 & -7.1 & 15.1 \\
\hline Exceptional financing & 26.1 & 24.3 & 18.2 & 17.5 & -40.5 \\
\hline Debt rescheduling & 0.0 & 0.0 & 0.0 & 0.0 & 0.0 \\
\hline Debt payments arrears (reduction -) & 26.1 & 24.3 & 18.2 & 17.5 & -40.5 \\
\hline \multicolumn{6}{|l|}{ Memorandum items: } \\
\hline Gross official reserves (at end of period) & 78.9 & 70.8 & 72.6 & 79.6 & 64.5 \\
\hline Current account (in percent of GDP) & -1.5 & -2.1 & -1.7 & -6.5 & -2.7 \\
\hline
\end{tabular}

Sources: C.A.R. authorities; Bank of Central African States (BEAC); and Fund staff estimates.

${ }^{1}$ Based on definitions consistent with the IMF's Balance of Payments Manual, 5th edition. 
Table 24. Central African Republic: Composition of Exports, 2002-06

\begin{tabular}{|c|c|c|c|c|c|}
\hline & 2002 & 2003 & 2004 & 2005 & $\begin{array}{r}2006 \\
\text { Est. }\end{array}$ \\
\hline & \multicolumn{5}{|c|}{$\begin{array}{l}\text { (Value in billions of CFA francs; volume in thousands of tons; } \\
\text { unit values in CFA francs per kilogram, unless otherwise indicated) }\end{array}$} \\
\hline Total exports, value & 102.3 & 74.4 & 66.7 & 67.5 & 82.4 \\
\hline \multicolumn{6}{|l|}{ Diamonds } \\
\hline Value & 36.3 & 28.6 & 27.8 & 32.8 & 32.7 \\
\hline Volume $^{1}$ & 414.8 & 329.6 & 354.2 & 383.3 & 415.5 \\
\hline Unit value ${ }^{2}$ & 87,600 & 86,200 & 78,400 & 85,600 & 78,660 \\
\hline \multicolumn{6}{|l|}{ Coffee $^{3}$} \\
\hline Value & 1.0 & 0.8 & 1.0 & 0.9 & 0.8 \\
\hline Volume & 5.7 & 2.0 & 3.8 & 2.5 & 2.6 \\
\hline Unit value & 179.6 & 358.0 & 271.3 & 369.8 & 297.4 \\
\hline \multicolumn{6}{|l|}{ Wood products } \\
\hline Value & 52.0 & 34.6 & 28.8 & 25.8 & 39.9 \\
\hline Volume $^{4}$ & 381.1 & 275.7 & 239.4 & 197.3 & 263.5 \\
\hline Unit value $^{5}$ & 130.4 & 121.2 & 120.1 & 131.0 & 151.5 \\
\hline \multicolumn{6}{|l|}{ Cotton } \\
\hline Value & 6.4 & 0.6 & 1.8 & 1.1 & 0.7 \\
\hline Volume & 12.3 & 0.9 & 2.9 & 2.3 & 1.4 \\
\hline Unit value & 520.7 & 621.2 & 612.3 & 473.9 & 494.8 \\
\hline \multicolumn{6}{|l|}{ Tobacco } \\
\hline Value & 0.1 & 0.1 & 0.1 & 0.1 & 0.1 \\
\hline Volume & 0.1 & 0.1 & 0.1 & 0.1 & 0.1 \\
\hline Unit value & 962 & 825 & 529.3 & 512.0 & 516.1 \\
\hline \multicolumn{6}{|l|}{ Miscellaneous } \\
\hline Value & 6.5 & 9.8 & 7.3 & 6.7 & 8.2 \\
\hline
\end{tabular}

Sources: C.A.R. authorities; Bank of Central African States (BEAC); and Fund staff estimates.

${ }^{1}$ In thousands of carats.

${ }^{2}$ In CFA francs per carat.

${ }^{3}$ Includes reexports.

${ }^{4}$ In thousands of cubic meters.

${ }^{5}$ In CFA francs per cubic meter. 
Table 25. Central African Republic: Export, Import Indices and Terms of Trade, 2002-06 1/

\begin{tabular}{|c|c|c|c|c|c|}
\hline & 2002 & 2003 & 2004 & 2005 & $\begin{array}{r}2006 \\
\text { Est. }\end{array}$ \\
\hline & \multicolumn{5}{|c|}{$(\text { Indices, } 1995=100)^{1}$} \\
\hline \multicolumn{6}{|l|}{ Exports $^{2}$} \\
\hline Value & 110.3 & 80.2 & 71.9 & 72.7 & 88.8 \\
\hline Volume & 124.8 & 92.0 & 90.6 & 83.3 & 95.8 \\
\hline Unit value & 88.4 & 87.2 & 79.4 & 87.4 & 92.7 \\
\hline \multicolumn{6}{|l|}{ Imports } \\
\hline Value & 92.6 & 75.5 & 86.4 & 99.9 & 117.5 \\
\hline Volume & 69.9 & 61.0 & 70.6 & 76.3 & 85.0 \\
\hline Unit value & 132.5 & 123.9 & 122.4 & 130.9 & 138.3 \\
\hline Terms of trade & 66.7 & 70.4 & 64.9 & 66.8 & 67.0 \\
\hline & \multicolumn{5}{|c|}{ 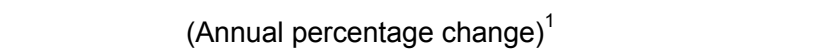 } \\
\hline \multicolumn{6}{|l|}{ Exports $^{2}$} \\
\hline Value & -1.7 & -27.3 & -10.3 & 1.1 & 22.1 \\
\hline Volume & 1.3 & -26.3 & -1.6 & -8.1 & 15.1 \\
\hline Unit value & -3.0 & -1.4 & -8.8 & 10.0 & 6.1 \\
\hline \multicolumn{6}{|l|}{ Imports } \\
\hline Value & 6.7 & -18.5 & 14.3 & 15.7 & 17.6 \\
\hline Volume & 9.9 & -12.8 & 15.7 & 8.2 & 11.3 \\
\hline Unit value & -2.9 & -6.5 & -1.2 & 6.9 & 5.7 \\
\hline Terms of trade & -0.1 & 5.5 & -7.7 & 2.8 & 0.4 \\
\hline
\end{tabular}

Sources: C.A.R. authorities; Bank of Central African States (BEAC); and Fund staff estimates.

${ }^{1}$ Based on unit values in CFA francs.

${ }^{2}$ Includes reexports. 
Table 26. Central African Republic: Balance of Payments-Selected Components of Services, Income, and Transfers, 2002-06

\begin{tabular}{|c|c|c|c|c|c|}
\hline & 2002 & 2003 & 2004 & 2005 & $\begin{array}{r}2006 \\
\text { Est. }\end{array}$ \\
\hline & \multicolumn{5}{|c|}{ (In billions of CFA francs) } \\
\hline Services (net) & -52.2 & -36.0 & -30.1 & -39.6 & -37.0 \\
\hline Credit & 10.3 & 15.0 & 22.0 & 22.4 & 26.0 \\
\hline Freight and insurance & 0.3 & 0.2 & 0.4 & 0.4 & 0.5 \\
\hline Other transport & 0.7 & 1.7 & 2.0 & 2.4 & 2.4 \\
\hline Travel & 2.1 & 2.6 & 4.1 & 2.5 & 4.0 \\
\hline Other private services & 1.3 & 1.9 & 2.9 & 2.5 & 2.5 \\
\hline Government services & 5.9 & 8.6 & 12.6 & 14.6 & 16.6 \\
\hline Debit & -62.5 & -51.0 & -52.1 & -62.0 & -63.0 \\
\hline Freight and insurance & -28.1 & -19.1 & -20.6 & -24.2 & -31.7 \\
\hline Petroleum products & -3.5 & -3.3 & -7.6 & -7.6 & -11.2 \\
\hline Other freight and insurance & -24.6 & -15.8 & -13.0 & -16.6 & -20.5 \\
\hline Other transport & -9.9 & -7.0 & -7.4 & -7.9 & -1.4 \\
\hline Travel & -20.5 & -19.1 & -19.1 & -22.0 & -22.2 \\
\hline Other private services & -3.8 & -5.6 & -4.8 & -7.1 & -6.9 \\
\hline Public administration services & -0.2 & -0.1 & -0.1 & -0.8 & -0.8 \\
\hline Income (net) & -6.1 & -1.4 & -0.9 & -1.1 & -2.1 \\
\hline Credit & 3.7 & 4.1 & 5.1 & 5.2 & 5.5 \\
\hline Compensation of employees & 3.4 & 4.1 & 5.1 & 5.2 & 5.3 \\
\hline Investment income & 0.3 & 0.0 & 0.0 & 0.0 & 0.2 \\
\hline Debit & -9.8 & -5.5 & -6.0 & -6.3 & -7.6 \\
\hline Compensation of employees & 0.0 & -0.1 & -0.1 & -0.1 & -0.6 \\
\hline Investment income & -9.8 & -5.4 & -5.9 & -6.2 & -7.0 \\
\hline Interest on public sector debt ${ }^{1}$ & -7.5 & -5.1 & -5.6 & -3.7 & -4.5 \\
\hline Public debt (budget concept) & -7.3 & -5.0 & -5.5 & -3.6 & -4.2 \\
\hline IMF & -0.1 & -0.1 & -0.1 & -0.1 & -0.2 \\
\hline Private sector & -2.3 & -0.3 & -0.3 & -2.5 & -2.5 \\
\hline Current transfers (net) & 28.6 & 16.8 & 30.5 & 16.2 & 42.2 \\
\hline Private (net) & 1.6 & -1.4 & 3.5 & 1.7 & 1.7 \\
\hline Official (net) & 27.0 & 18.2 & 27.0 & 14.5 & 40.5 \\
\hline
\end{tabular}

Sources: C.A.R. authorities; Bank of Central African States (BEAC); and Fund staff estimates.

${ }^{1}$ Scheduled interest, including IMF charges. 


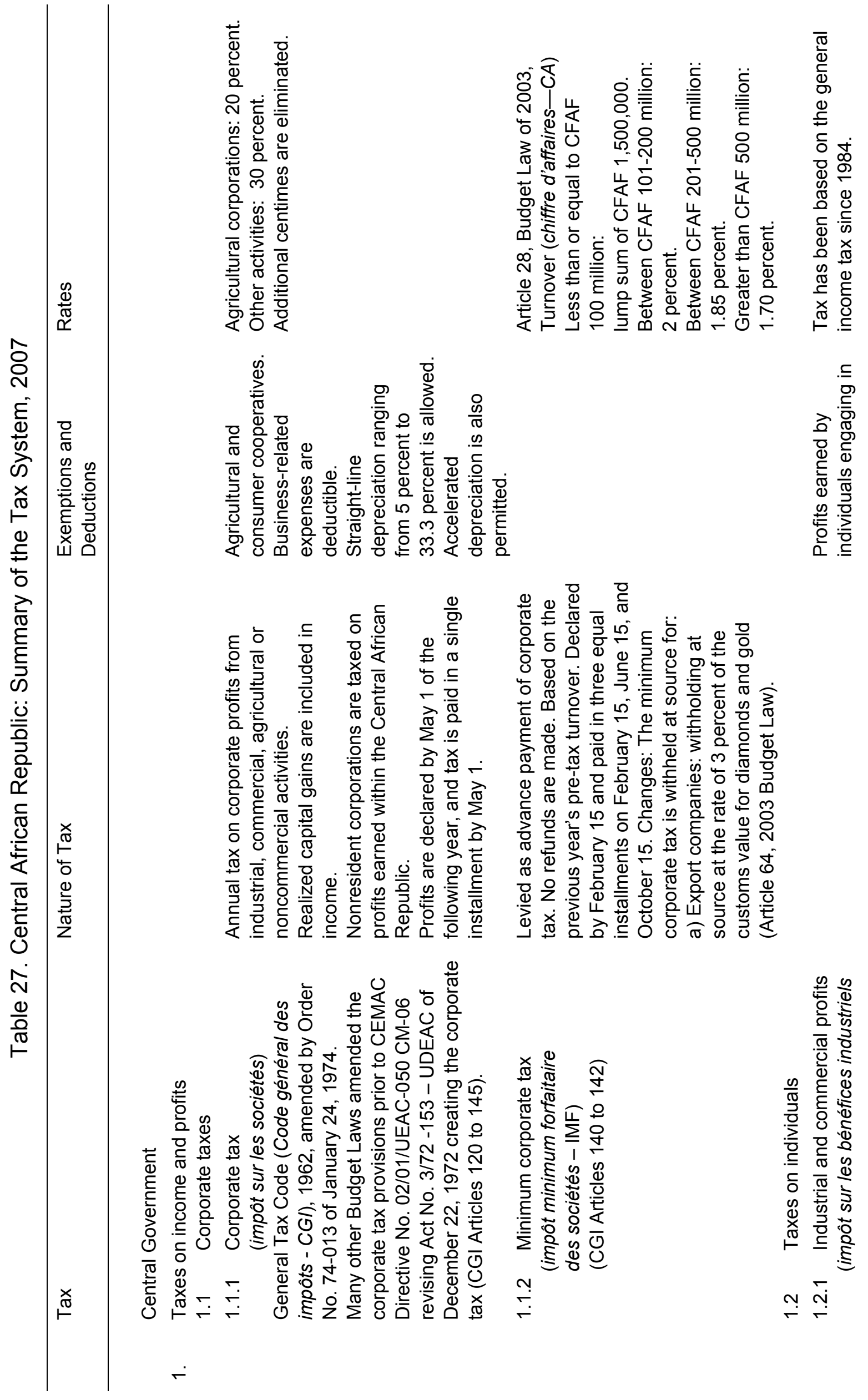




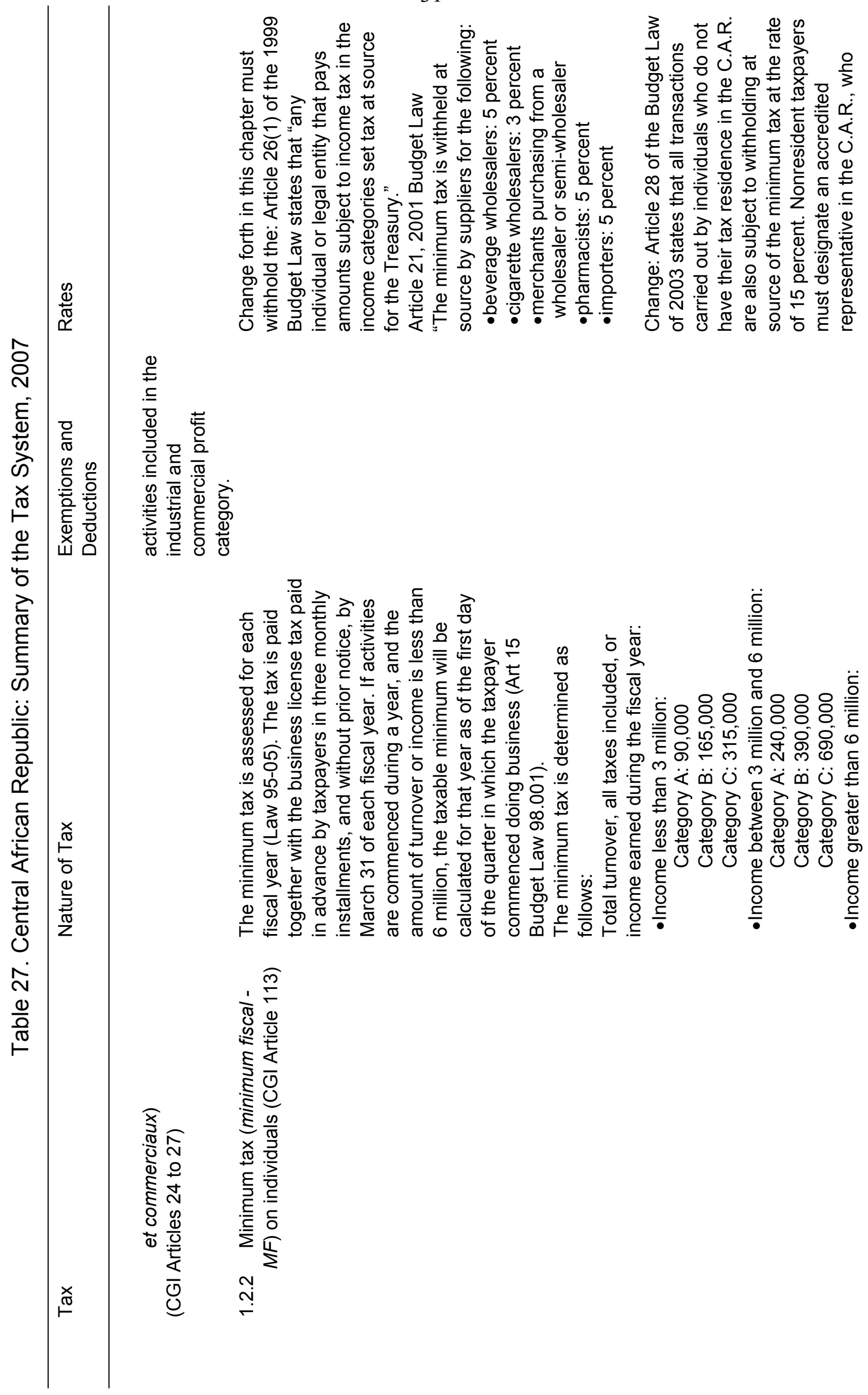




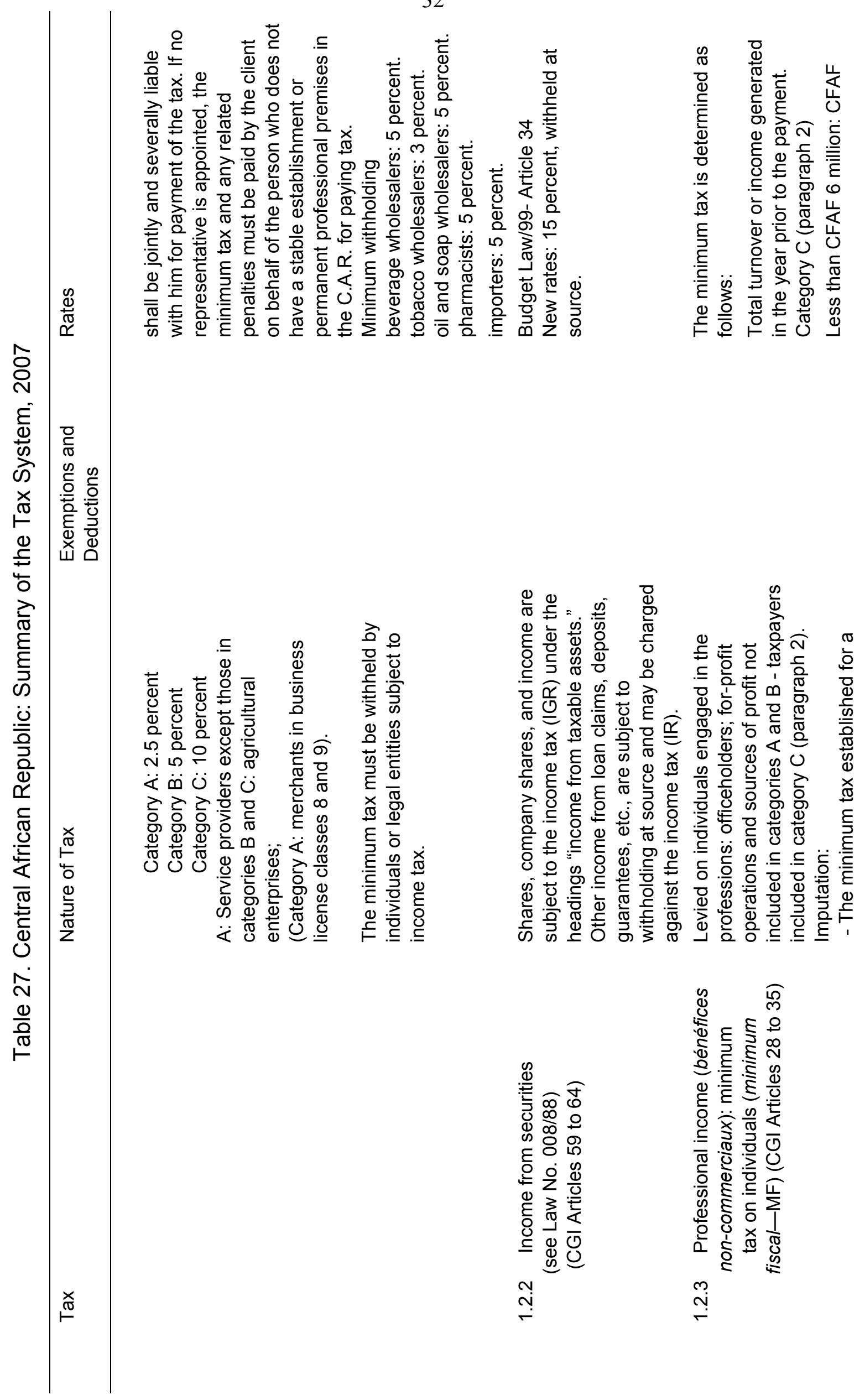




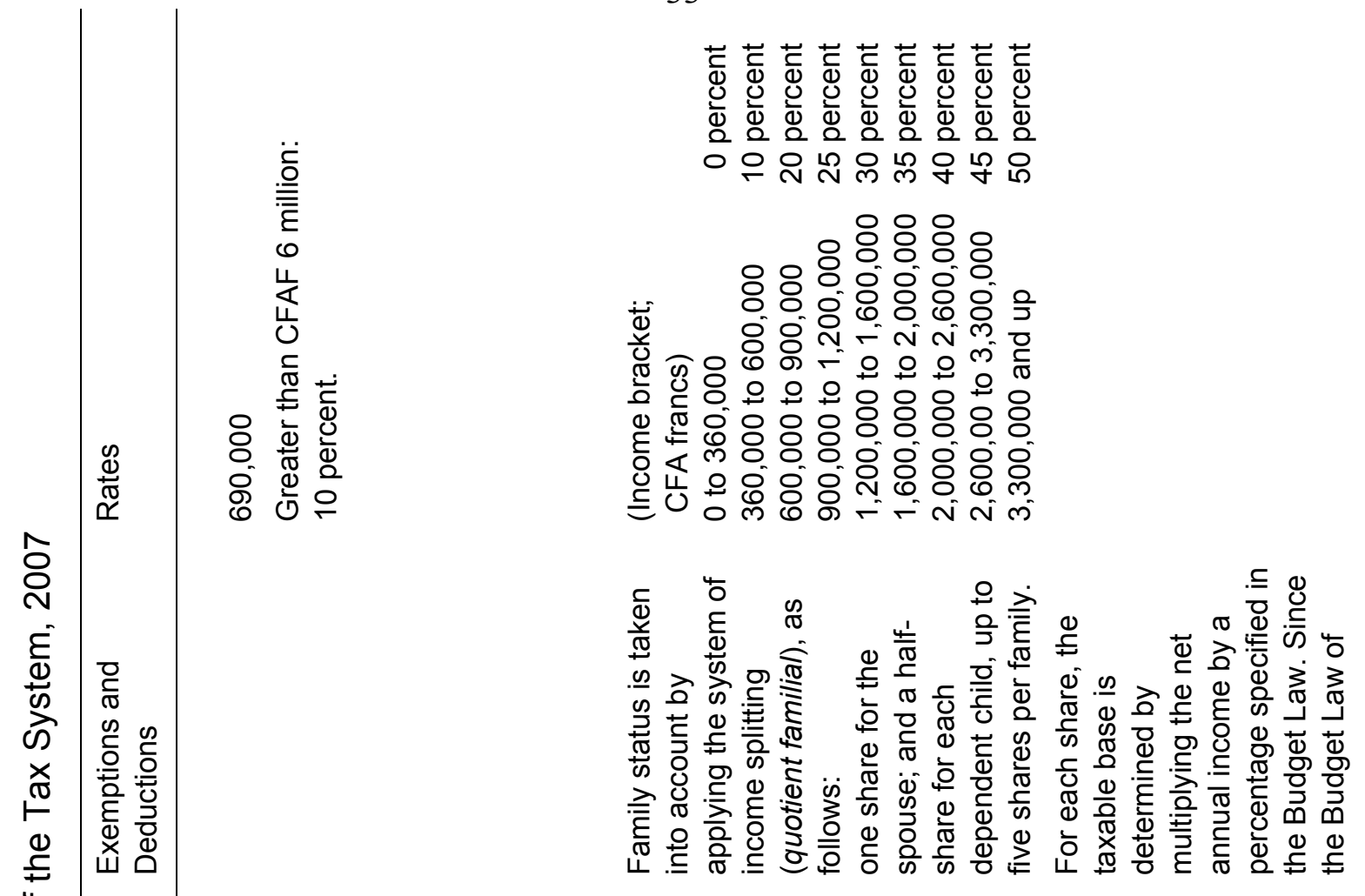




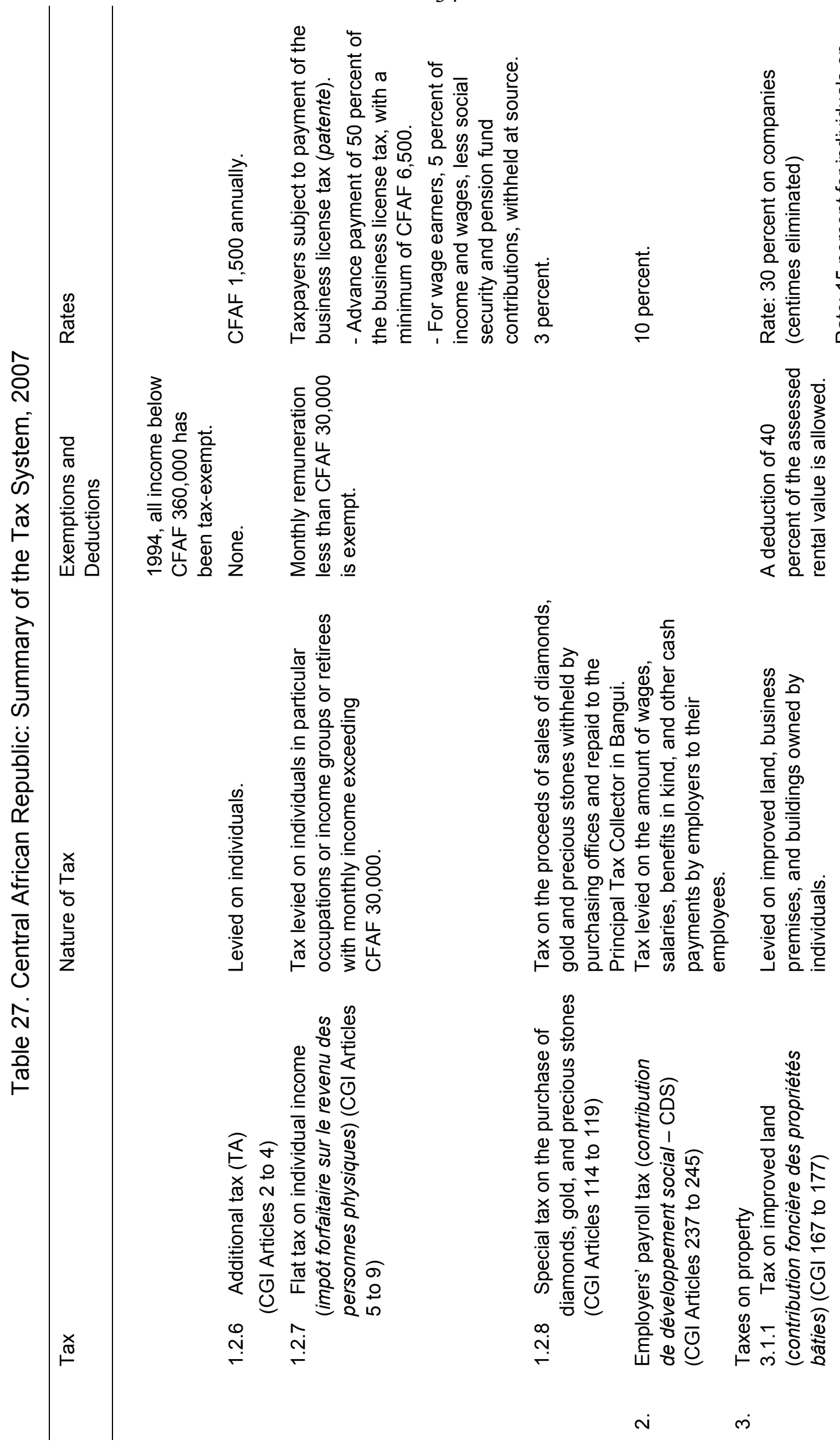


35

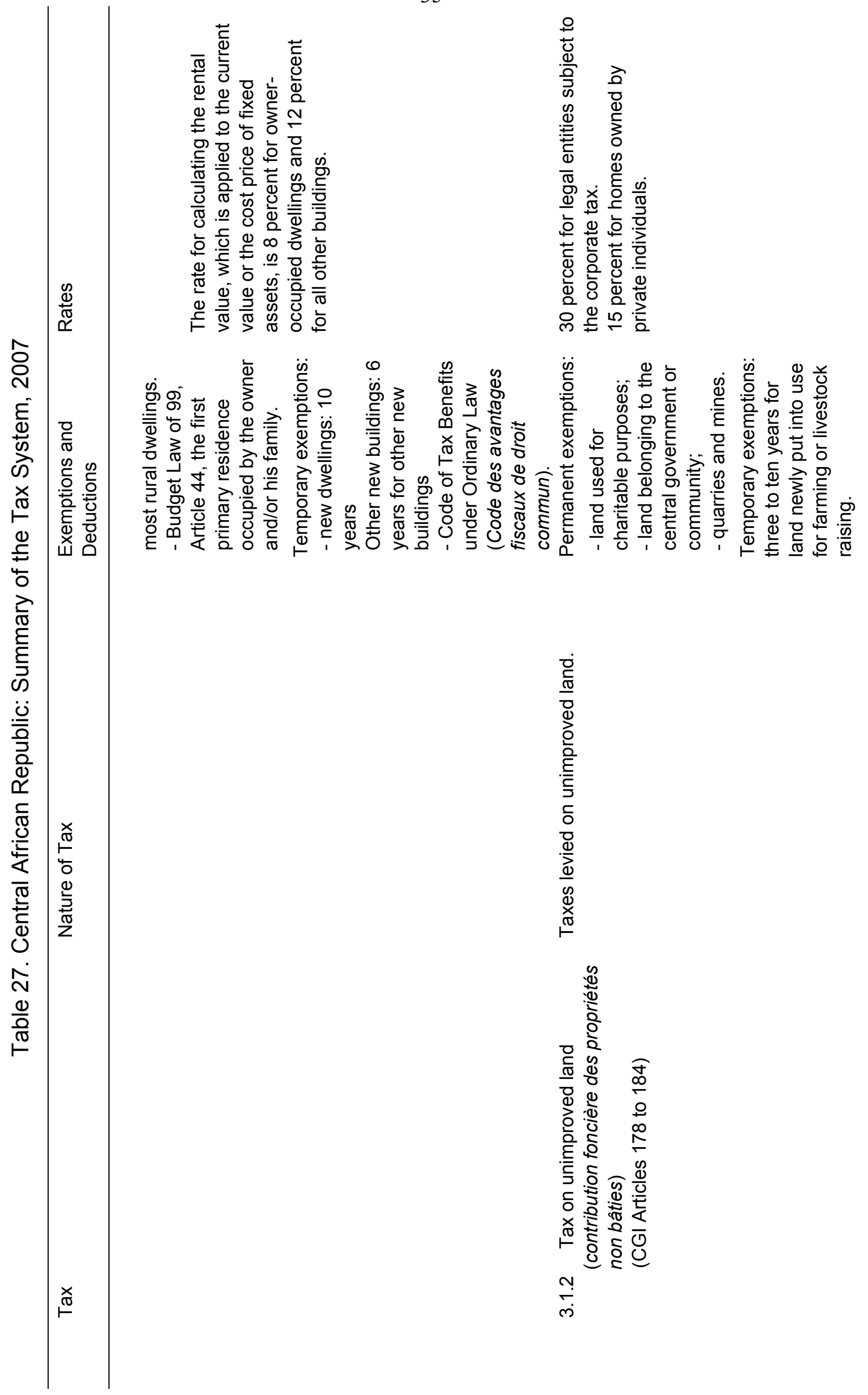


36

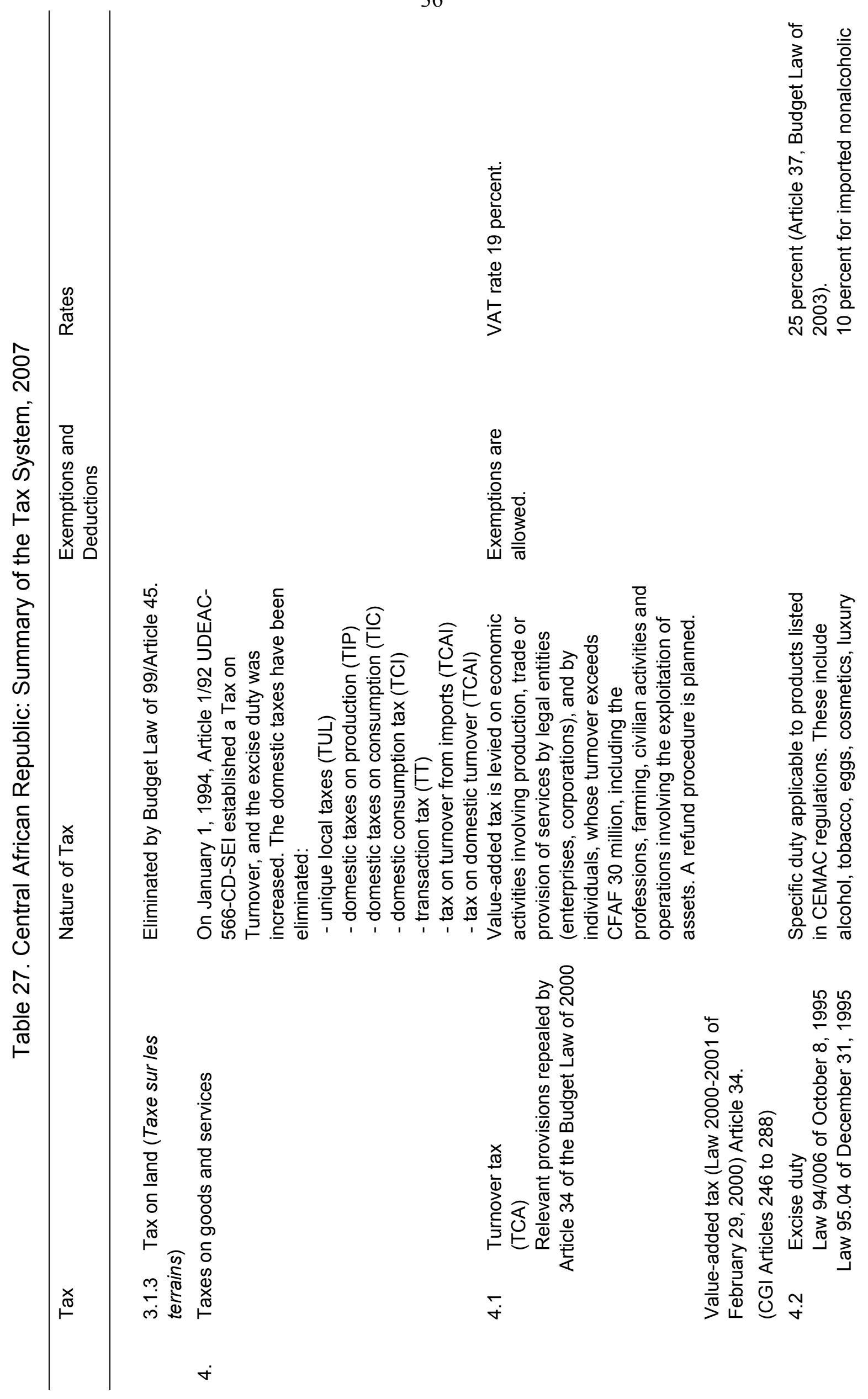




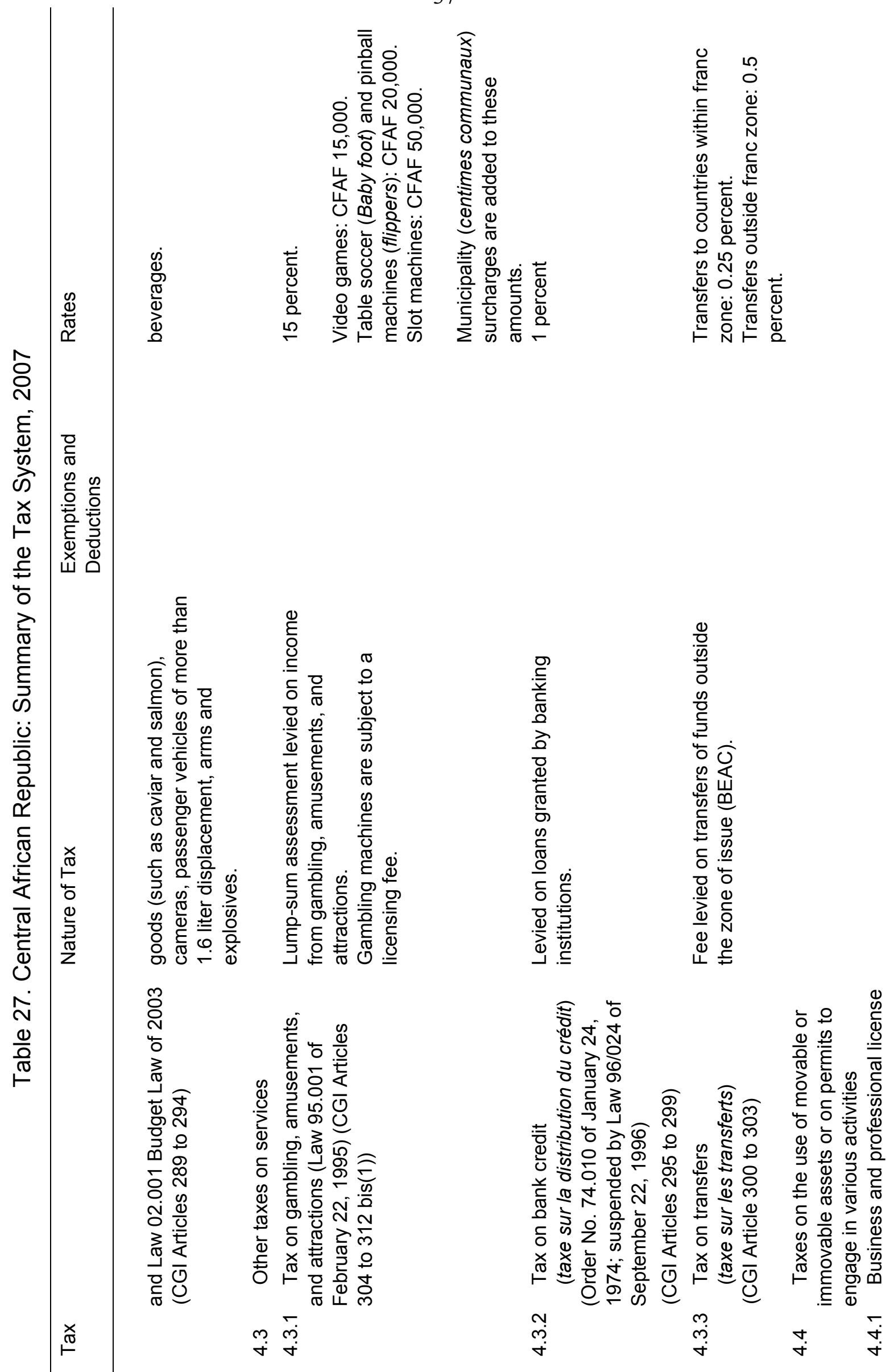




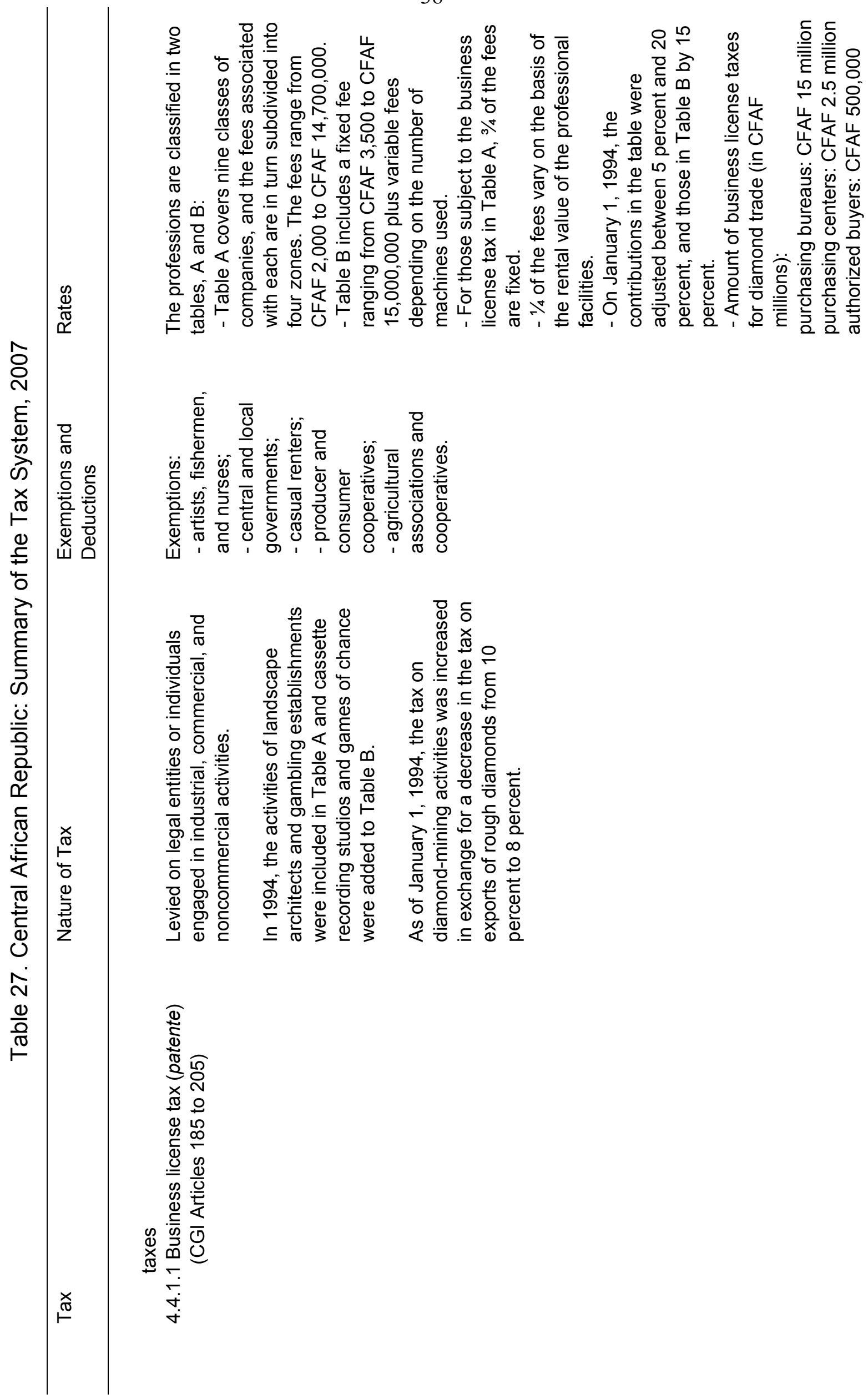




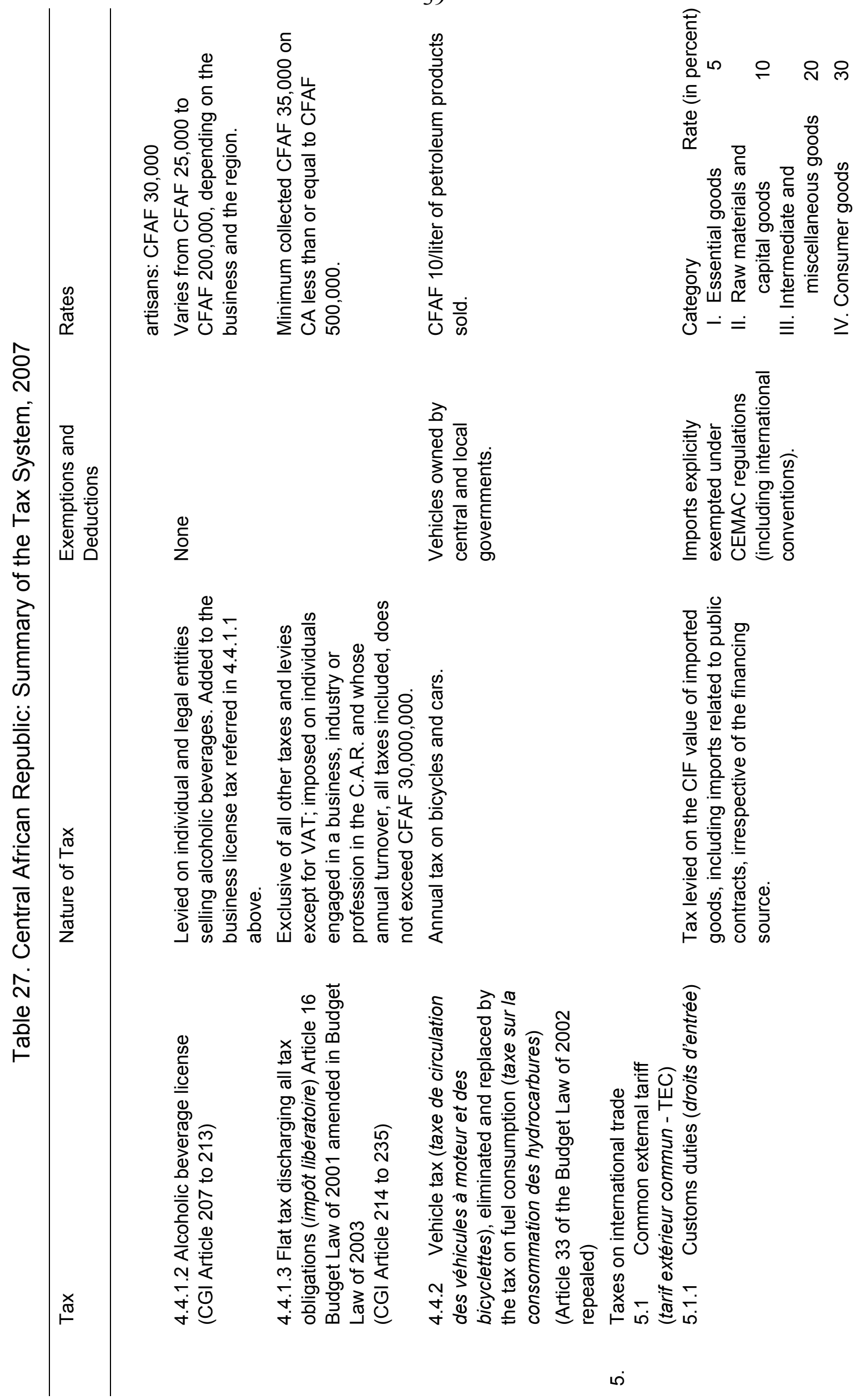



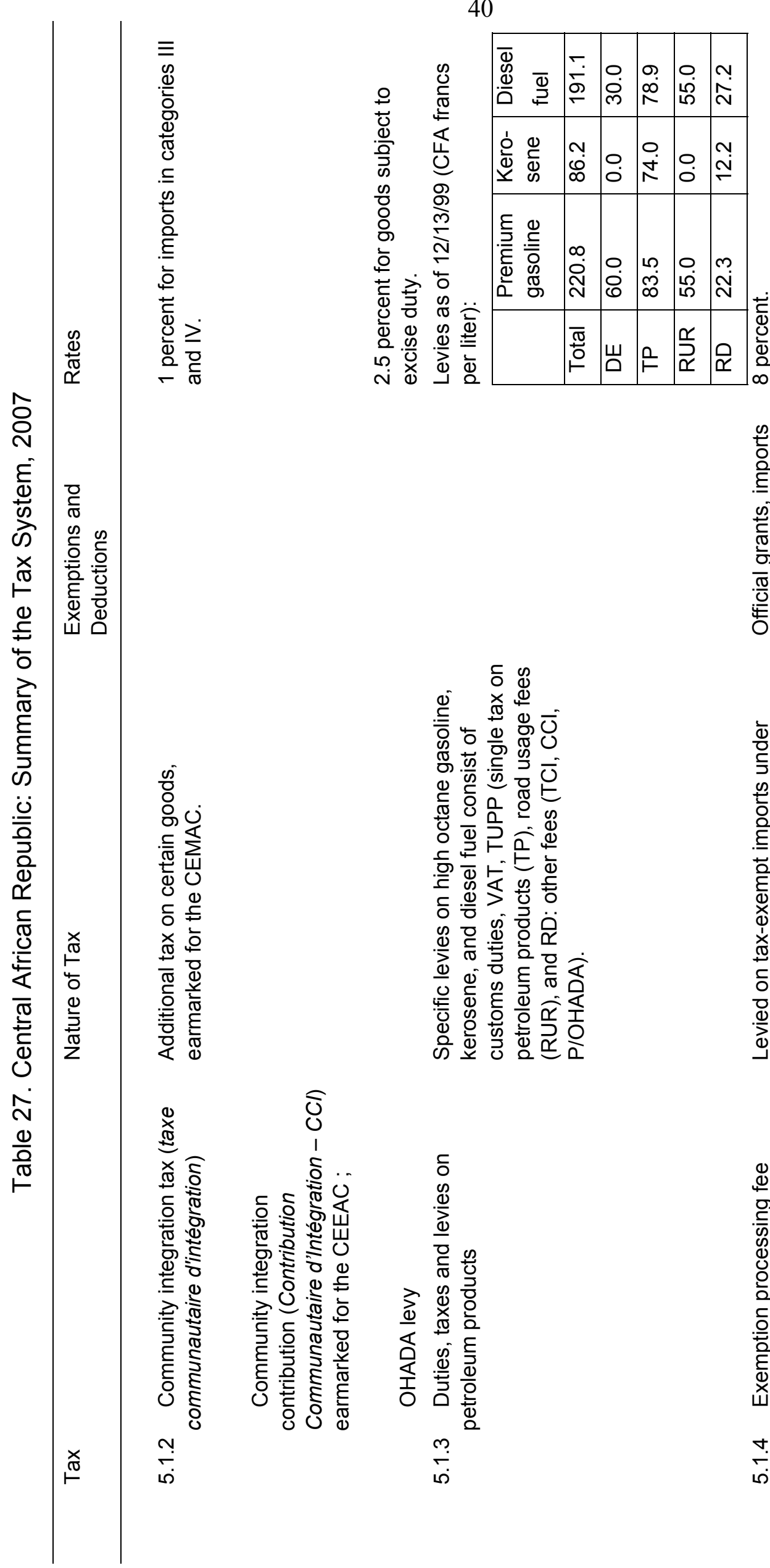

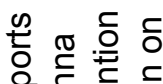

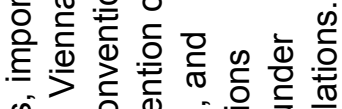
s. 㐘

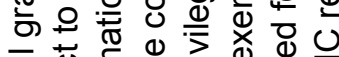
त

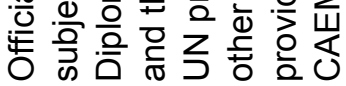
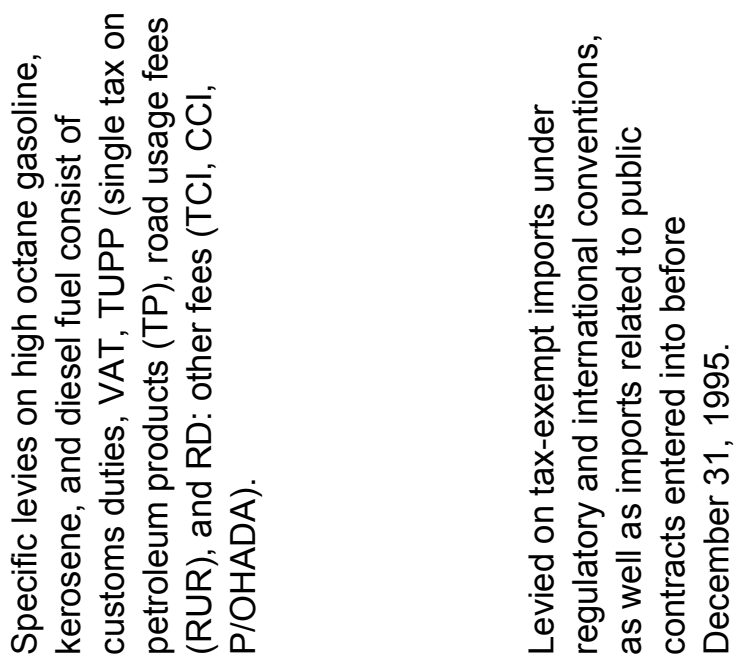


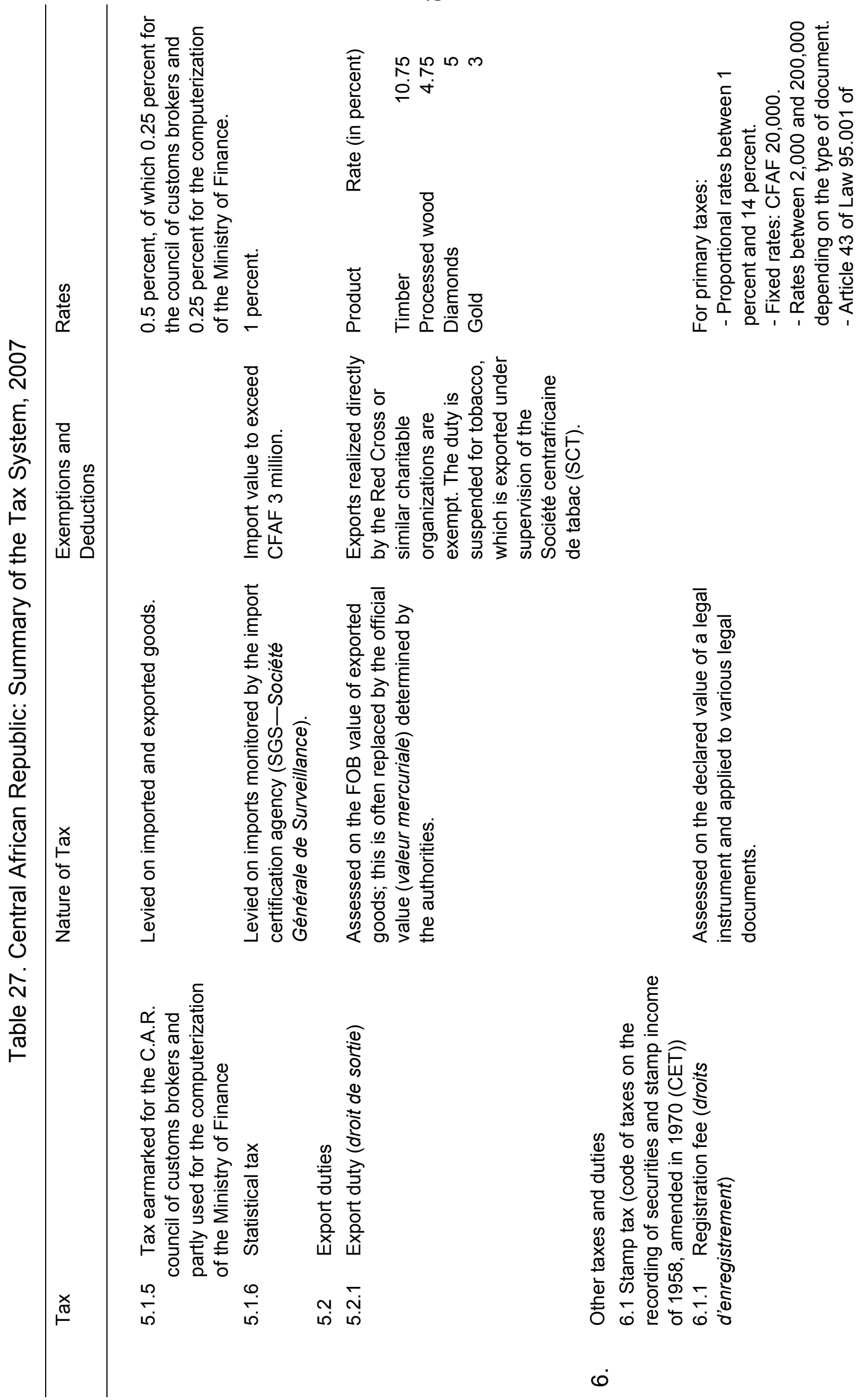




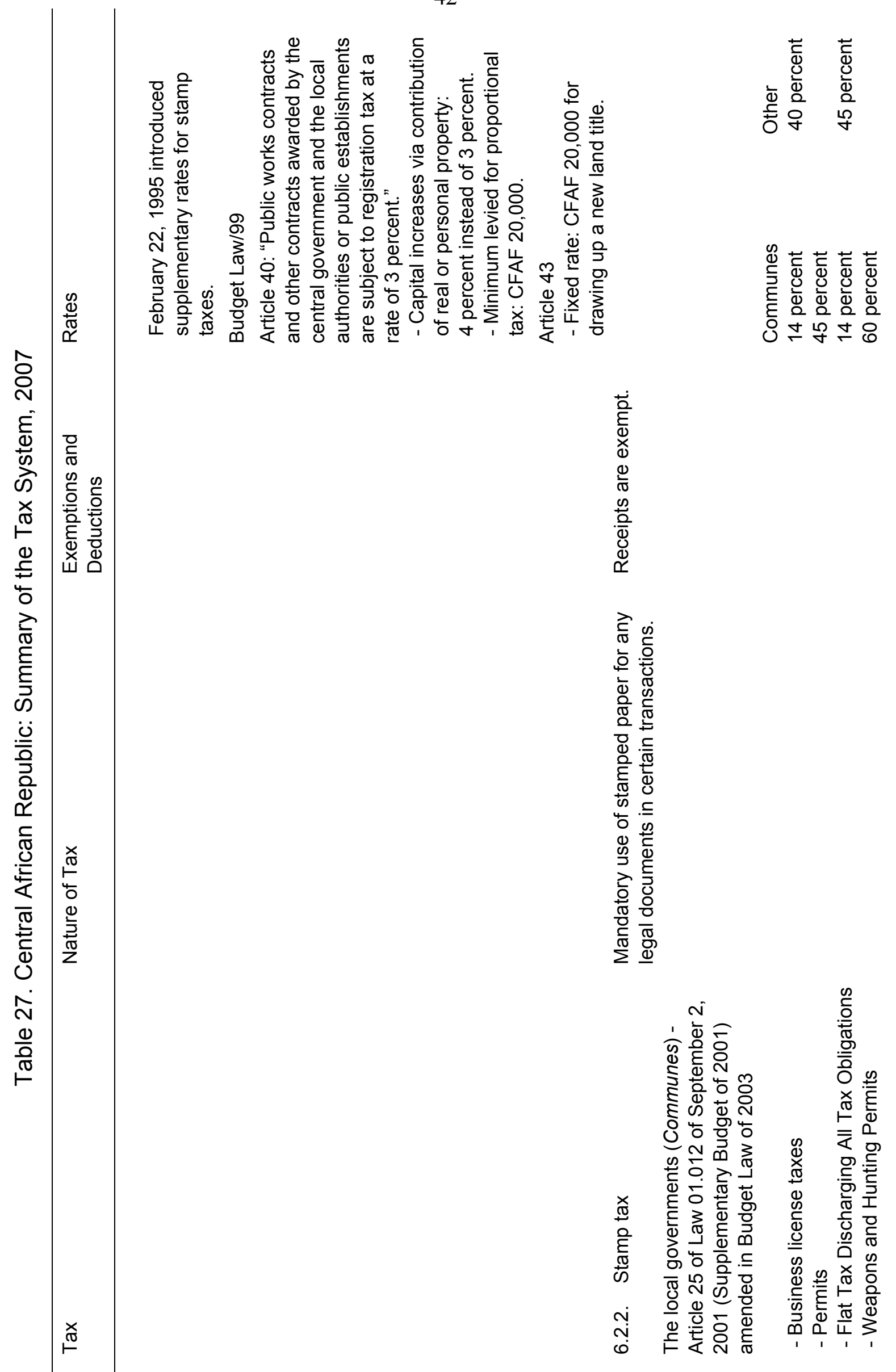




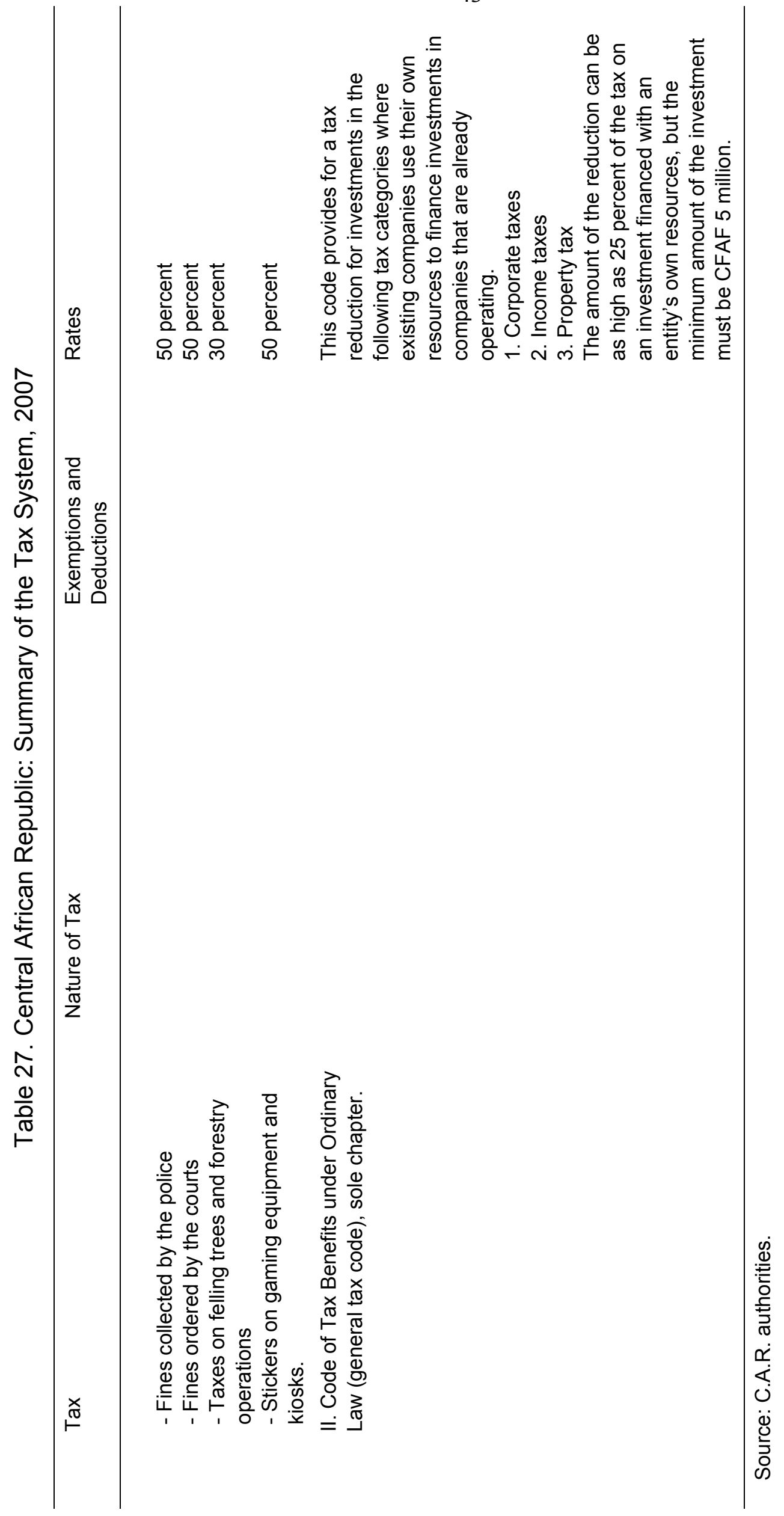

